\title{
Well posedness conditions for Bimodal Piecewise Affine Systems
}

\author{
Gökhan Şahan ${ }^{\mathrm{a}, *, 1}$, Vasfi Eldem ${ }^{\mathrm{b}}$ \\ ${ }^{a}$ Izmir Institute of Technology, Department of Mathematics, Urla, Izmir, Turkey \\ ${ }^{\mathrm{b}}$ Okan University, Department of Basic Sciences in Engineering, Akfirat-Tuzla İstanbul, Turkey
}

\section{A R T I C L E I N F O}

\section{Article history:}

Received 23 September 2014

Received in revised form

14 May 2015

Accepted 9 June 2015

Available online 1 July 2015

\section{Keywords:}

Switched systems

Bimodal systems

Well posedness

Existence and uniqueness

Carathéodory solution

Nonsmooth systems

\begin{abstract}
A B S T R A C T
This paper considers well-posedness (the existence and uniqueness of the solutions) of Bimodal Piecewise Affine Systems in $\mathbb{R}^{n}$. It is assumed that both modes are observable, but only one of the modes is in observable canonical form. This allows the vector field to be discontinuous when the trajectories change mode. Necessary and sufficient conditions for well-posedness are given as a set of algebraic conditions and sign inequalities. It is shown that these conditions induce a joint structure for the system matrices of the two modes. This structure can be used for the classification of well-posed bimodal piecewise affine systems. Furthermore, it is also shown that, under certain conditions, well-posed Bimodal Piecewise Affine Systems in $\mathbb{R}^{n}$ may have one or two equilibrium points or no equilibrium points.
\end{abstract}

(C) 2015 Elsevier B.V. All rights reserved.

\section{Introduction}

Switched systems consist of subsystems (modes) and a switching signal that organizes which subsystem drives the overall system. Piecewise Linear Systems (PLS) are a subclass of switched systems where subsystems are linear, time invariant and the switching is autonomous (state dependent). Since PLS are driven by both continuous and discrete dynamics (switching), they exhibit a rich dynamic behavior. The reader may refer to the books by Filippov [1], di Bernardo et al. [2] and tutorial papers by Cortes et al. [3], and Goebel et al. [4], which contain many examples demonstrating the dynamic behavior of PLS. A general treatment of switched systems is given by Liberzon [5].

Bimodal Piecewise Linear Systems (BPLS) and Bimodal Piecewise Affine Systems (BPAS) are special subclasses of PLS where there are only two subsystems. Subsystems in BPLS are homogeneous ( $\dot{\mathbf{x}}=\mathbf{A}_{i} \mathbf{x}, i=1,2$ ), whereas in BPAS a constant vector is added to the vector field $\left(\dot{\mathbf{x}}=\mathbf{A}_{i} \mathbf{x}+\mathbf{b}_{i}, i=1,2\right)$.

BPLS and BPAS attracted considerable attention in the literature lately. This is basically due to the fact that these systems can be

\footnotetext{
* Corresponding author.

E-mail addresses: gsahan@iyte.edu.tr (G. Șahan),vasfi.eldem@okan.edu.tr (V. Eldem).

1 Currently he is a visiting scholar at Coordinated Science Laboratory, University of Illinois at Urbana Champaign, IL, USA.
}

used for modeling a rich variety of mechanical, electrical, biological and engineering systems. As a consequence, BPLS and BPAS are investigated by many authors in the context of stability, stabilizability and control in the literature (see, for instance, [6-10]). In addition to this, BPAS turns out to be an appropriate tool to understand nonlinear phenomena. For instance, BPAS version of the well known traveling wave equation model of Michelson System [11], the Wien-bridge oscillator of [12], a biological network model by Imura et al. [13], are examples of nonlinear systems modeled as BPAS.

Well-posedness simply means existence and uniqueness of solutions. If the vector field is assumed to be continuous, then for each initial condition in $\mathbb{R}^{n}$, there is a unique and continuously differentiable solution of PLS which resolves the issue of wellposedness. The literature contains many papers which investigate stability [14,6], controllability and observability [15,7,9] under this assumption. If the vector field is discontinuous, then dynamic behavior of PLS cannot be represented by continuously differentiable solutions and well-posedness become a central issue in PLS. There are various solution structures for many classes of systems with discontinuous vector fields and the details and examples can be found in the survey papers by Georgescu et al. [16] and Cortes [3] and in the book [17]. An interesting dynamic behavior encountered in PLS is the existence of trajectories which change mode infinite number of times in a finite time interval. This paradoxical behavior is called Zeno behavior and investigated in a series of papers $[15,18,19]$. Moreover, well-posedness is also an essential issue in problems such as stability, stabilizability and feedback 
control. Along this line, the reader may refer to Example 13 in [20], where well-posedness conditions guarantee global asymptotic stability of a BPLS in $\mathbb{R}^{2}$.

Well-posedness of BPLS in $\mathbb{R}^{n}$ is first solved by Imura and van der Schaft [21] in the context of Carathéodory solutions. Imura [22] investigated well-posedness of BPAS with control inputs in $\mathbb{R}^{n}$ and presented a classification of well-posed feedback systems in $\mathbb{R}^{n}$. The papers by Çamlıbel et al. [15], by Çamlıbel [18], and by Thuan and Çamlıbel [19] have also provided important contributions on the Zeno behavior of well-posed PLS.

In this paper, we consider well-posedness problem of BPAS in $\mathbb{R}^{n}$ where both modes are observable. We assume, without loss of any generality, that only the system matrix of one of the modes is in observable canonical form. This allows for discontinuities of the vector field. It is shown that necessary and sufficient conditions of well-posedness given in this paper induce a special structure on the matrix of the subsystem which is not in observable canonical form. This leads to a joint structure for the two modes, which holds for both BPLS and BPAS. This structure can be checked easily and be used for classification of well-posed BPAS and BPLS. Finally, at the end of the paper, we exploit the relation between the conditions of well-posedness and the equilibrium points of BPAS and provide the conditions under which BPAS have one or two or no equilibrium points. Comparison of the results presented here with the results given in the literature (such as [21-23]) are given in Remarks 3.33.5 in the paper.

\section{Preliminaries}

In this section we investigate well-posedness conditions of the following BPAS

$\Sigma_{0}:\left\{\begin{array}{ll}\dot{\mathbf{x}}=\mathbf{A}_{1} \mathbf{x}+\mathbf{b}_{1}, & \text { if } \mathbf{c}^{T} \mathbf{x} \geq \mathbf{0} \\ \dot{\mathbf{x}}=\mathbf{A}_{2} \mathbf{x}+\mathbf{b}_{2}, & \text { if } \mathbf{c}^{T} \mathbf{x} \leq \mathbf{0}\end{array}\right\}$

where $\mathbf{A}_{i} \in \mathbb{R}^{n \times n}, \mathbf{b}_{i}, \mathbf{c} \in \mathbb{R}^{n}$. The following definition is crucial in our approach.

Definition 2.1. The observability index of a linear time invariant system is the smallest integer $k$ such that $\operatorname{rank}\left(\mathcal{O}_{k}^{i}\right)=\operatorname{rank}\left(\mathcal{O}_{k+1}^{i}\right)$ where $\mathcal{O}_{k}^{i}:=\left[\begin{array}{lllll}\mathbf{c} & \mathbf{A}_{i}^{T} \mathbf{c} & . . & . \cdot & \left(\mathbf{A}_{i}^{k-1}\right)^{T} \mathbf{c}\end{array}\right]^{T}$ for $i=1,2$. If $k=n$, then the pair $\left(\mathbf{c}^{T}, \mathbf{A}_{i}\right)$ is said to be observable.

In view of this definition, we assume that: "The pairs $\left(\mathbf{c}^{T}, \mathbf{A}_{1}\right)$ and $\left(\mathbf{c}^{T}, \mathbf{A}_{2}\right)$ are observable and the pair $\left(\mathbf{c}^{T}, \mathbf{A}_{2}\right)$ is in observable canonical form".

Note that assuming $\left(\mathbf{c}^{T}, \mathbf{A}_{2}\right)$ to be in observable canonical form does not cause any loss of generality since $\mathbf{A}_{1}$ is completely free. In view of this assumption, the components of $\Sigma_{0}$ are given as follows.

$\mathbf{A}_{1}=\left[\begin{array}{ccc}a_{11} & \cdots & a_{1 n} \\ \vdots & \ddots & \vdots \\ a_{n 1} & \ldots & a_{n n}\end{array}\right], \quad \mathbf{A}_{2}=\left[\begin{array}{ccccc}k_{1} & k_{2} & \cdots & \cdots & k_{n} \\ 1 & 0 & \cdots & \cdots & 0 \\ 0 & 1 & 0 & \cdots & \vdots \\ \vdots & \ddots & \ddots & \ddots & \vdots \\ 0 & \cdots & 0 & 1 & 0\end{array}\right]$,

$\mathbf{b}_{i}=\left[\begin{array}{c}b_{i 1} \\ \vdots \\ b_{i n}\end{array}\right], \quad i=1,2$ and $\mathbf{c}^{T}=\left[\begin{array}{llll}0 & \cdots & 0 & 1\end{array}\right]$.

For well-posedness of $\Sigma_{0}$, we only consider the solutions in the sense of Carathéodory as defined below. This definition is taken from [18].
Definition 2.2. An absolutely continuous function $\mathbf{x}: \mathbb{R} \rightarrow \mathbb{R}^{n}$ is said to be a solution of $\Sigma_{0}$ for the initial condition $\mathbf{x}_{0}$ in the sense of

1. Carathéodory if $\mathbf{x}(0)=\mathbf{x}_{0}$ and $\mathbf{x}(t)$ satisfies (1) for almost all $t \in \mathbb{R}$,

2. Forward Carathéodory for the initial state $\mathbf{x}_{0}$, if it is a solution in the sense of Carathéodory, and for each $t_{0} \geq 0$, there exists $\epsilon^{+}>0$ such that either $\dot{\mathbf{x}}=\mathbf{A}_{1} \mathbf{x}+\mathbf{b}_{1}$ and $\mathbf{c}^{T} \mathbf{x} \geq 0$, or $\dot{\mathbf{x}}=\mathbf{A}_{2} \mathbf{x}+\mathbf{b}_{2}$ and $\mathbf{c}^{T} \mathbf{x} \leq 0$, hold for all $t \in\left[t_{0}, t_{0}+\epsilon^{+}\right]$.

Definition 2.3. The system $\Sigma_{0}$ is said to be well-posed if for every initial state $\mathbf{x}(0)=\mathbf{x}_{0} \in \mathbb{R}^{n}$, there exists a unique forward Carathéodory solution $\mathbf{x}(t)$ of $\Sigma_{0}$.

Definition 2.4. Let $s_{i}, i=1,2$ denote the set of initial conditions $\mathbf{x}_{0} \in \mathbb{R}^{n}$ defined as follows: For each $\mathbf{x}_{0} \in s_{i}$ there exists $\epsilon_{i}>0$ and a forward Carathéodory solution with $\mathbf{x}(0)=\mathbf{x}_{0}$ and $\dot{\mathbf{x}}=\mathbf{A}_{i} \mathbf{x}+\mathbf{b}_{i}$ such that $\mathbf{x}(t) \in \varsigma_{i}$ for all $t \in\left[0, \epsilon_{i}\right]$. Then, we say that the solution with $\mathbf{x}(0)=\mathbf{x}_{0} \in \wp_{i}$ smoothly continues in mode $i$.

This definition is consistent with Definition 2.3 in [21]. In order to show that $s_{i}$ 's $(i=1,2)$ are nonempty, let $\mathscr{H}$ denote the switching hyperplane defined as follows.

$\mathscr{H}:=\left\{\mathbf{x} \in \mathbb{R}^{n} \mid \mathbf{c}^{T} \mathbf{x}=0\right\}$.

Note that $\mathscr{H}$ is a subspace of dimension $n-1$ which divides $\mathbb{R}^{n}$ into two open half spaces defined as

$\mathscr{H}^{+}:=\left\{\mathbf{x} \in \mathbb{R}^{n} \mid \mathbf{c}^{T} \mathbf{x}>0\right\} \quad$ and $\quad \mathscr{H}^{-}:=\left\{x \in \mathbb{R}^{n} \mid \mathbf{c}^{T} \mathbf{x}<0\right\}$.

In view of the theory of differential equations, for any initial condition $\mathbf{x}_{0} \in \mathscr{H}^{+}\left(\mathbf{x}_{0} \in \mathscr{H}^{-}\right)$, there exists $\varepsilon^{+}>0\left(\varepsilon^{-}>0\right)$ and a forward Carathéodory solution $\mathbf{x}(t)$ such that $\mathbf{x}(0)=$ $\mathbf{x}_{0}, \mathbf{c}^{T} \mathbf{x}(t)>0$ and $\dot{\mathbf{x}}=\mathbf{A}_{1} \mathbf{x}+\mathbf{b}_{1}\left(\mathbf{c}^{T} \mathbf{x}(t)<0\right.$ and $\left.\dot{\mathbf{x}}=\mathbf{A}_{2} \mathbf{x}+\mathbf{b}_{2}\right)$ for all $t \in\left[0, \varepsilon^{+}\right]\left(t \in\left[0, \varepsilon^{-}\right]\right)$. Since only one of the modes is active, the solution is unique. This implies that $s_{1}$ and $s_{2}$ are nonempty. More precisely, $\mathscr{H}^{+} \subset \wp_{1}$ and $\mathscr{H}^{-} \subset \wp_{2}$. If $\mathbf{x}_{0} \in \mathscr{H}$, then we cannot claim uniqueness of solutions since both modes are allowed to be active on $\mathcal{H}$. Therefore, the problem of well-posedness reduces to the characterization of the sets $\delta_{i} \cap \mathscr{H}$, for $i=1,2$. In order to resolve this problem, we present below two Lemmas from $[21,22]$. These Lemmas give necessary and sufficient conditions for wellposedness of BPLS and BPAS, respectively.

Lemma 2.5 ([21, Lemma 2.1]). Let $\mathbf{b}_{1}=\mathbf{b}_{2}=\mathbf{0}$. Then the following are equivalent.

1. $\Sigma_{0}$ is well-posed

2. From every initial state $\mathbf{x}_{0} \in \mathbb{R}^{n}$, smooth continuation is possible in only one of the two modes, except for the case when the solutions for both modes are the same in some time interval.

Lemma 2.6 ([22, Lemma 2.2-(i)]). $\quad \Sigma_{0}$ is well-posed if and only if $\varsigma_{1} \cup \varsigma_{2}=\mathbb{R}^{n}$ and for every $\mathbf{x}_{0} \in \varsigma_{1} \cap \varsigma_{2}$ the solutions according to both modes are the same in some time interval.

We should remark here that in [22, Lemma 2.2-(i)], C-wellposedness is used instead of well-posedness. Since C-wellposedness is the same as well-posedness given in Definition 2.3, we simply use well-posedness here.

Remark 2.7. Note that the conditions given in [22, Lemma 2.2-(i)] are also valid for BPLS. This follows from the fact that $s_{1} \cap \delta_{2}$ is a single point in $\mathbb{R}^{n}$ and this point is the origin, i.e., the equilibrium point of well-posed BPLS. Hence, the solutions in both modes are the same. It will be shown in the sequel that $\delta_{1} \cap \delta_{2}$ is also a single point for a well-posed BPAS. This point may also be the equilibrium point of BPAS (see item 5 of Corollary 3.2). 
In view of Definition 2.3 and Lemma's given above, wellposedness is equivalent to the fact that the derivatives with respect to $t$ of the solutions, i.e. $\frac{d\left(c^{T} x\right)}{d t}$, for both modes must have the same sign at any $\mathbf{x}_{0} \in \mathscr{H}$. We can formalize this observation by using the classification given in [24, pp. 58] as follows. BPAS,

Let $\mathbf{x}_{0} \in \mathscr{H}$. Then, the following hold for a well-posed BPLS or

- Either $\mathbf{c}^{T}\left(\mathbf{A}_{i} \mathbf{x}_{0}+\mathbf{b}_{i}\right)>0$ for $i=1,2$ or $\mathbf{c}^{T}\left(\mathbf{A}_{i} \mathbf{x}_{0}+\mathbf{b}_{i}\right)<0$ for $i=1,2$.

- The case $\mathbf{c}^{T}\left(\mathbf{A}_{1} \mathbf{x}_{0}+\mathbf{b}_{1}\right)>0$ and $\mathbf{c}^{T}\left(\mathbf{A}_{2} \mathbf{x}_{0}+\mathbf{b}_{2}\right)<0$ is not allowed because it implies that there are two solutions smoothly continuing in two modes and this contradicts uniqueness.

- The case $\mathbf{c}^{T}\left(\mathbf{A}_{1} \mathbf{x}_{0}+\mathbf{b}_{1}\right)<0$ and $\mathbf{c}^{T}\left(\mathbf{A}_{2} \mathbf{x}_{0}+\mathbf{b}_{2}\right)>0$ is not allowed because it implies that there are no solutions in the sense of Carathéodory.

Nonlinear version of the framework given above is previously used for the investigation of well-posedness of bimodal systems with nonlinear subsystems in [25].

\section{Main results}

We now present the main result of the paper.

Theorem 3.1. Consider the piecewise affine system $\Sigma_{0}$ defined by Eqs. (1) and (2). The system is well-posed if and only if the following hold.

(1) The structure of $\mathbf{A}_{1}$ is such that $a_{i j}=0$ if $i=3,4, \ldots, n$ and $j=1,2, \ldots, i-2$, or equivalently the following hold $\bigcap_{r=0}^{s} \operatorname{ker}\left(\mathbf{c}^{T} \mathbf{A}_{1}^{r}\right)=\bigcap_{r=0}^{s} \operatorname{ker}\left(\mathbf{c}^{T} \mathbf{A}_{2}^{r}\right)$ for $s=1,2, \ldots, n-1$.

(2) The entries of $\mathbf{A}_{1}$ which are below the diagonal are positive i.e., $a_{i+1, i}>0$ for $i=1,2, \ldots, n-1$.

(3) The relation between $\mathbf{b}_{1}$ and $\mathbf{b}_{2}$ is as follows.

$$
\left[\begin{array}{cccc}
a_{21} & a_{22} & \cdots & a_{2, n-1} \\
0 & a_{32} & \ddots & \vdots \\
\vdots & \ddots & \ddots & \vdots \\
0 & \cdots & 0 & a_{n, n-1}
\end{array}\right]\left[\begin{array}{c}
b_{22} \\
\vdots \\
b_{2 n}
\end{array}\right]=\left[\begin{array}{c}
b_{12} \\
\vdots \\
b_{1 n}
\end{array}\right] .
$$

(4) The following equation hold for the entries of $\mathbf{b}_{1}$ and $\mathbf{b}_{2}$.

$$
\begin{aligned}
& a_{n, n-1} a_{n-1, n-2} \ldots a_{21}\left(b_{11}-\sum_{j=1}^{n-1} a_{1 j} b_{2, j+1}\right) \\
& =b_{21}-\sum_{j=1}^{n-1} k_{j} b_{2, j+1} .
\end{aligned}
$$

Note that items 3 and 4 of Theorem 3.1 give the algebraic relations between the affine terms of modes, while items 1 and 2 impose a special structure on $\mathbf{A}_{1}$ as shown below.

$$
\mathbf{A}_{1}=\left[\begin{array}{ccccc}
a_{11} & a_{12} & \cdots & a_{1, n-1} & a_{1 n} \\
a_{21} & a_{22} & \cdots & a_{2, n-1} & a_{2 n} \\
0 & a_{32} & \ddots & \vdots & \vdots \\
\vdots & \ddots & \ddots & \vdots & \vdots \\
0 & \cdots & 0 & a_{n, n-1} & a_{n n}
\end{array}\right]
$$

If we partition both system matrices, their joint structure (induced by well-posedness conditions) can be displayed as follows.

$\mathbf{A}_{1}=\left[\begin{array}{cc}\mathbf{y}_{1}^{T} & a_{1 n} \\ \mathbf{A}_{11} & \mathbf{z}_{1}\end{array}\right], \quad$ and $\quad \mathbf{A}_{2}=\left[\begin{array}{cc}\mathbf{y}_{2}^{T} & k_{n} \\ \mathbf{I} & \mathbf{0}\end{array}\right]$,

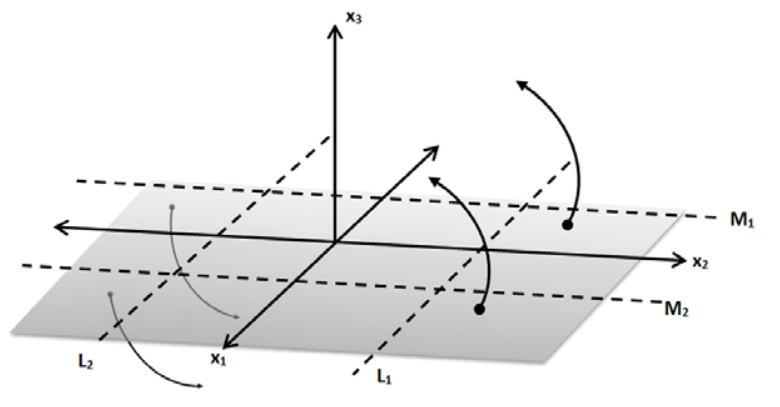

Fig. 1. Plane geometry when $L_{1} \neq L_{2}$ and $M_{1} \neq M_{2}$.

where $\mathbf{y}_{1}, \mathbf{y}_{2}$, and $\mathbf{z}_{1}$ are in $\mathbb{R}^{n-1}$ and $\mathbf{A}_{11} \in \mathbb{R}^{(n-1) \times(n-1)}$ as defined below.

$\mathbf{A}_{11}:=\left[\begin{array}{cccc}a_{21} & a_{22} & \cdots & a_{2, n-1} \\ 0 & a_{32} & \ddots & \vdots \\ \vdots & \ddots & \ddots & \vdots \\ 0 & \cdots & 0 & a_{n, n-1}\end{array}\right], \quad \mathbf{y}_{1}:=\left[\begin{array}{c}a_{11} \\ a_{12} \\ \vdots \\ a_{1, n-1}\end{array}\right]$,

$\mathbf{z}_{1}:=\left[\begin{array}{c}a_{2 n} \\ \vdots \\ \vdots \\ a_{n n}\end{array}\right], \quad \mathbf{y}_{2}:=\left[\begin{array}{c}k_{1} \\ k_{2} \\ \vdots \\ k_{n-1}\end{array}\right]$.

The proof of Theorem 3.1 is technical and it is given in the Appendix. In the sequel, we first give an example to motivate the proof of Theorem 3.1.

Example 3.2. Consider the following BPAS.

$\mathbf{A}_{i}=\left[\begin{array}{ccc}\mathfrak{a}_{i} & \mathfrak{b}_{i} & \mathfrak{c}_{i} \\ 1 & 0 & 0 \\ 0 & 1 & 0\end{array}\right], \quad i=1,2$,

$\mathbf{b}_{1}=\left[\begin{array}{c}2 \\ 1 \\ -3\end{array}\right], \quad \mathbf{b}_{2}=\left[\begin{array}{c}1 \\ -2 \\ 2\end{array}\right], \quad \mathbf{c}=\left[\begin{array}{l}0 \\ 0 \\ 1\end{array}\right]$

where $\mathfrak{a}_{i}, \mathfrak{b}_{i}$ and $\mathfrak{c}_{i}$ are arbitrary real numbers. Let us define the lines $L_{1}$ and $L_{2}$ in $\mathscr{H}:=\operatorname{ker} \mathbf{c}^{T}$ as follows.

$L_{1}=\left\{\mathbf{x}_{0} \in \mathscr{H} \mid \mathbf{x}_{0}=\left[\begin{array}{lll}\gamma_{1} & 3 & 0\end{array}\right]^{T}, \gamma_{1} \in \mathbb{R}\right\}$,

$L_{2}=\left\{\mathbf{x}_{0} \in \mathscr{H} \mid \mathbf{x}_{0}=\left[\begin{array}{lll}\gamma_{1} & -2 & 0\end{array}\right]^{T}, \gamma_{1} \in \mathbb{R}\right\}$,

where $\gamma_{1}$ is an arbitrary real number (these lines are depicted in Fig. 1). Note that if $\mathbf{x}_{0} \in L_{1}\left(\mathbf{x}_{0} \in L_{2}\right)$ then $\mathbf{c}^{T}\left(\mathbf{A}_{1} \mathbf{x}_{0}+\mathbf{b}_{1}\right)=$ $0\left(\mathbf{c}^{T}\left(\mathbf{A}_{2} \mathbf{x}_{0}+\mathbf{b}_{2}\right)=0\right)$. Let $\mathbf{x}_{0}=\left[\begin{array}{lll}\gamma_{1} & \gamma_{2} & 0\end{array}\right]^{T}$ be an arbitrary initial condition in $\mathcal{H}$. Note that if $\gamma_{2}>3$, then it follows that the first derivatives with respect to $t$ of the solutions for both modes are $\mathbf{c}^{T}\left(\mathbf{A}_{1} \mathbf{x}_{0}+\mathbf{b}_{1}\right)=\gamma_{2}-3>0$ and $\mathbf{c}^{T}\left(\mathbf{A}_{2} \mathbf{x}_{0}+\mathbf{b}_{2}\right)=\gamma_{2}+2>$ 0 . Similarly, if $\gamma_{2}<-2$, then it follows that $\mathbf{c}^{T}\left(\mathbf{A}_{1} \mathbf{x}_{0}+\mathbf{b}_{1}\right)<$ 0 and $\mathbf{c}^{T}\left(\mathbf{A}_{2} \mathbf{x}_{0}+\mathbf{b}_{2}\right)<0$. Consequently, for each initial condition $\mathbf{x}_{0} \in \mathscr{H}$ where $\gamma_{2}>3\left(\gamma_{2}<-2\right)$, there exists a unique forward Carathéodory solution which smoothly continues in first mode (second mode). However, if $-2<\gamma_{2}<3$, then $\mathbf{c}^{T}\left(\mathbf{A}_{2} \mathbf{x}_{0}+\mathbf{b}_{2}\right)>0$ and $\mathbf{c}^{T}\left(\mathbf{A}_{1} \mathbf{x}_{0}+\mathbf{b}_{1}\right)<0$. This implies that there are no solutions in the sense of Carathéodory. Hence, the lines $L_{1}$ and $L_{2}$ should be the same in order to guarantee the existence of a unique forward Carathéodory solution for each $\mathbf{x}_{0} \in \mathscr{H}$ and $\mathbf{x}_{0} \notin L_{1}\left(=L_{2}\right)$.

Let us change $\mathbf{b}_{2}$ as $\mathbf{b}_{2}=\left[\begin{array}{lll}1 & -2 & -3\end{array}\right]^{T}$ so that $L_{1}=L_{2}$ and define $\mathscr{H}_{1}$ as follows.

$$
\begin{aligned}
\mathscr{H}_{1} & :=\left\{\mathbf{x}_{0} \in \mathscr{H} \mid \mathbf{c}^{T}\left(\mathbf{A}_{1} \mathbf{x}_{0}+\mathbf{b}_{1}\right)=\mathbf{c}^{T}\left(\mathbf{A}_{2} \mathbf{x}_{0}+\mathbf{b}_{2}\right)=0\right\}, \\
& =\left\{\mathbf{x}_{0} \in \mathscr{H} \mid \mathbf{x}_{0}=\left[\begin{array}{lll}
\gamma_{1} & 3 & 0
\end{array}\right]^{T}\right\} .
\end{aligned}
$$




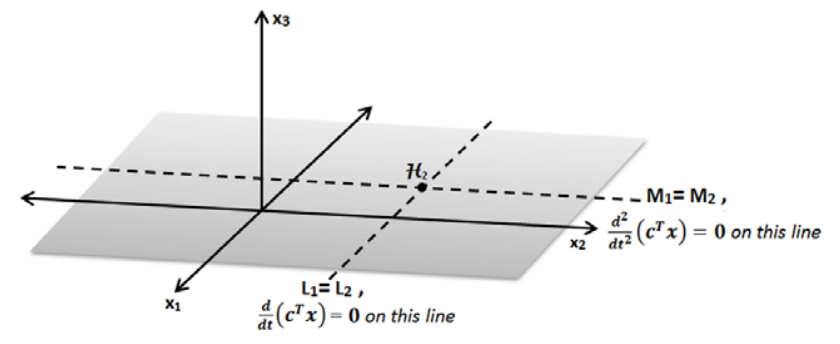

Fig. 2. Plane geometry for well-posed system.

Note that $\mathscr{H}_{1}$ divides $\mathscr{H}$ into two affine spaces (each with dimension two) which can be described as follows.

$\mathscr{H}_{1}^{+}:=\left\{\mathbf{x}_{0} \in \mathscr{H} \mid \mathbf{c}^{T}\left(\mathbf{A}_{1} \mathbf{x}_{0}+\mathbf{b}_{1}\right)>0\right.$ and $\left.\mathbf{c}^{T}\left(\mathbf{A}_{2} \mathbf{x}_{0}+\mathbf{b}_{2}\right)>0\right\}$,

$\mathscr{H}_{1}^{-}:=\left\{\mathbf{x}_{0} \in \mathscr{H} \mid \mathbf{c}^{T}\left(\mathbf{A}_{1} \mathbf{x}_{0}+\mathbf{b}_{1}\right)<0\right.$ and $\left.\mathbf{c}^{T}\left(\mathbf{A}_{2} \mathbf{x}_{0}+\mathbf{b}_{2}\right)<0\right\}$.

Let us now consider the following lines, $M_{1} \in \mathscr{H}$ and $M_{2} \in$ $\mathcal{H}$, where $\mathbf{c}^{T}\left(\mathbf{A}_{1}^{2} \mathbf{x}_{0}+\mathbf{A}_{1} \mathbf{b}_{1}\right)=0$ and $\mathbf{c}^{T}\left(\mathbf{A}_{2}^{2} \mathbf{x}_{0}+\mathbf{A}_{2} \mathbf{b}_{2}\right)=0$, respectively. These lines can be described as follows (depicted in Fig. 1).

$M_{1}=\left\{\mathbf{x}_{0} \in \mathscr{H} \mid \mathbf{x}_{0}=\left[\begin{array}{lll}-1 & \gamma_{2} & 0\end{array}\right]^{T}, \gamma_{2} \in \mathbb{R}\right\}$,

$M_{2}=\left\{\mathbf{x}_{0} \in \mathscr{H} \mid \mathbf{x}_{0}=\left[\begin{array}{lll}2 & \gamma_{2} & 0\end{array}\right]^{T}\right\}, \quad \gamma_{2} \in \mathbb{R}$

where $\gamma_{2}$ is an arbitrary real number. Note that $M_{1}$ and $M_{2}$ intersect $\mathscr{H}_{1}$ at $\gamma_{2}=3$. Then, consider an initial condition $\mathbf{x}_{0} \in \mathscr{H}$ defined as $\mathbf{x}_{0}:=\left[\begin{array}{lll}\gamma_{1} & 3 & 0\end{array}\right]^{T}$. Note that if $\gamma_{1}>2$, then it follows that the second derivatives of the trajectory are $\mathbf{c}^{T}\left(\mathbf{A}_{1}^{2} \mathbf{x}_{0}+\mathbf{A}_{1} \mathbf{b}_{1}\right)=$ $\gamma_{1}+1>0$ and $\mathbf{c}^{T}\left(\mathbf{A}_{2}^{2} \mathbf{x}_{0}+\mathbf{A}_{2} \mathbf{b}_{2}\right)=\gamma_{1}-2>0$. If $\gamma_{1}<-1$, then we get $\mathbf{c}^{T}\left(\mathbf{A}_{1}^{2} \mathbf{x}_{0}+\mathbf{A}_{1} \mathbf{b}_{1}\right)<0$ and $\mathbf{c}^{T}\left(\mathbf{A}_{2}^{2} \mathbf{x}_{0}+\mathbf{A}_{2} \mathbf{b}_{2}\right)<0$. Consequently, for each initial condition $\mathbf{x}_{0} \in \mathscr{H}_{1}$ where $\gamma_{1}>$ $2\left(\gamma_{1}<-1\right)$, there exists a unique forward Carathéodory solution which smoothly continues in first mode (second mode). However, if $-1<\gamma_{1}<2$ (corresponds to the points in between $M_{1}$ and $\left.M_{2}\right)$, then $\mathbf{c}^{T}\left(\mathbf{A}_{2}^{2} \mathbf{x}_{0}+\mathbf{A}_{2} \mathbf{b}_{2}\right)<0$ and $\mathbf{c}^{T}\left(\mathbf{A}_{1}^{2} \mathbf{x}_{0}+\mathbf{A}_{1} \mathbf{b}_{1}\right)>0$. This implies that there are two solutions in the sense of Carathéodory. One smoothly continues in $\varsigma_{1}$ and the other smoothly continues in $\varsigma_{2}$. Therefore, the lines $M_{1}$ and $M_{2}$ should be the same in order to guarantee the existence of unique forward Carathéodory solution for each $\mathbf{x}_{0} \in \mathscr{H}_{1}$ and $\mathbf{x}_{0} \notin M_{1}\left(=M_{2}\right)$.

Let us change $\mathbf{b}_{2}$ as $\mathbf{b}_{2}=\left[\begin{array}{lll}1 & 1 & -3\end{array}\right]^{T}$ so that $M_{1}=M_{2}$ and define $\mathscr{H}_{2}$ as follows.

$$
\begin{aligned}
\mathscr{H}_{2} & :=\left\{\mathbf{x}_{0} \in \mathscr{H}_{1} \mid \mathbf{c}^{T}\left(\mathbf{A}_{1}^{2} \mathbf{x}_{0}+\mathbf{A}_{1} \mathbf{b}_{1}\right)=\mathbf{c}^{T}\left(\mathbf{A}_{2}^{2} \mathbf{x}_{0}+\mathbf{A}_{2} \mathbf{b}_{2}\right)=0\right\}, \\
& =\left\{\mathbf{x}_{0} \in \mathscr{H}_{1} \mid \mathbf{x}_{0}=\left[\begin{array}{lll}
-1 & 3 & 0
\end{array}\right]^{T}\right\} .
\end{aligned}
$$

Along the same lines, $\mathscr{H}_{2}$ divides $\mathscr{H}_{1}$ into two half lines (each with dimension one) which can be described as follows.

$$
\begin{aligned}
\mathscr{H}_{2}^{+}:= & \left\{\mathbf{x}_{0} \in \mathscr{H}_{1} \mid \mathbf{c}^{T}\left(\mathbf{A}_{1}^{2} \mathbf{x}_{0}+\mathbf{A}_{1} \mathbf{b}_{1}\right)>0\right. \text { and } \\
& \left.\mathbf{c}^{T}\left(\mathbf{A}_{2}^{2} \mathbf{x}_{0}+\mathbf{A}_{2} \mathbf{b}_{2}\right)>0\right\}, \\
\mathscr{H}_{2}^{-}:= & \left\{\mathbf{x}_{0} \in \mathscr{H}_{1} \mid \mathbf{c}^{T}\left(\mathbf{A}_{1}^{2} \mathbf{x}_{0}+\mathbf{A}_{1} \mathbf{b}_{1}\right)<0\right. \text { and } \\
& \left.\mathbf{c}^{T}\left(\mathbf{A}_{2}^{2} \mathbf{x}_{0}+\mathbf{A}_{2} \mathbf{b}_{2}\right)<0\right\} .
\end{aligned}
$$

Note that $\mathscr{H}_{2}$ is a single point (depicted in Fig. 2) and with new $\mathbf{b}_{2}=\left[\begin{array}{lll}1 & 1 & -3\end{array}\right]^{T}$, the existence and uniqueness of solutions for each $\mathbf{x}_{0} \in \mathbb{R}^{3}$ is guaranteed, except for $\mathbf{x}_{0}^{*}=\left[\begin{array}{lll}-1 & 3 & 0\end{array}\right]^{T}=\mathscr{H}_{2}$.

Note that the third derivative of $\mathbf{c}^{T} \mathbf{x}(t)$ at $t=0$ where $\mathbf{x}(0)=$ $\mathbf{x}_{0}^{*}$, is easily calculated as follows.

$$
\left.\frac{d^{3}}{\mathrm{~d} t^{3}}\left(\mathbf{c}^{T} \mathbf{x}\right)\right|_{t=0}=\left\{\begin{array}{l}
\mathbf{c}^{T} \mathbf{A}_{1}^{3} \mathbf{x}_{0}^{*}+\mathbf{c}^{T} \mathbf{A}_{1}^{2} \mathbf{b}_{1} \\
\mathbf{c}^{T} \mathbf{A}_{2}^{3} \mathbf{x}_{0}^{*}+\mathbf{c}^{T} \mathbf{A}_{2}^{2} \mathbf{b}_{2}
\end{array}\right\}=\left\{\begin{array}{l}
2-\mathfrak{a}_{1}+3 \mathfrak{b}_{1} \\
1-\mathfrak{a}_{2}+3 \mathfrak{b}_{2}
\end{array}\right\} .
$$

The equality $2-\mathfrak{a}_{1}+3 \mathfrak{b}_{1}=1-\mathfrak{a}_{2}+3 \mathfrak{b}_{2}$ implies and implied by $\mathbf{A}_{1} \mathbf{x}_{0}^{*}+\mathbf{b}_{1}=\mathbf{A}_{2} \mathbf{x}_{0}^{*}+\mathbf{b}_{2}$. Consequently, these conditions are necessary and sufficient for the existence of a unique forward Carathéodory solution starting from $\mathbf{x}_{0}^{*}$.

Remark 3.3. Smooth continuation sets $\left(\wp_{1}, \wp_{2}\right)$ are characterized by lexicographic inequalities in [21,22]. These inequalities also imply that the vector field of both modes have a certain structure on $\mathcal{H}$, but this structure is displayed implicitly for BPLS by Theorems 4.1 and 4.2 in [21]. Theorem 4.2 of [21] gives the necessary and sufficient conditions for well-posedness of BPLS. For the case where $\left(\mathbf{c}^{T}, \mathbf{A}_{1}\right)$ and $\left(\mathbf{c}^{T}, \mathbf{A}_{2}\right)$ are observable, these conditions reduce to the existence of a lower left triangular matrix $\mathbf{M}$ with positive diagonal entries such that $\mathcal{O}_{n}^{1}=\mathbf{M} \mathcal{O}_{n}^{2}\left(\mathcal{O}_{k}^{i}\right.$ is the observability matrix given in Definition 2.1). It can be easily shown (by setting $b_{1}=b_{2}=0$ in the proof of Theorem 3.1) that this condition is equivalent to first and second items of Theorem 3.1. Thus, in our approach $\mathbf{M}$ arises naturally within the structure of $\mathbf{A}_{1}$. Moreover, starting with only one of the modes in observable canonical form, we arrive at a joint structure for a well-posed BPLS given by Eqs. (5)-(7). This structure can be checked by simple inspection and used for an explicit classification of well-posed BPLS. We should point out here that this result was first proven for BPLS in $\mathbb{R}^{3}$ in [8] and it was noted that these results can be extended to bimodal systems in $\mathbb{R}^{n}$.

Our main result, extends this result to well-posed BPAS and yields a joint structure for the triples $\left(\mathbf{c}^{T}, \mathbf{A}_{1}, \mathbf{b}_{1}\right)$ and $\left(\mathbf{c}^{T}, \mathbf{A}_{2}, \mathbf{b}_{2}\right)$ which can also be checked by simple inspection and used for classification of well-posed BPAS. Finally, we should also remark here that our main result can be easily extended to the case of unobservable subsystems, if the observability indices of both modes are equal. In this case, the joint structure of $\left(\mathbf{c}^{T}, \mathbf{A}_{1}, \mathbf{b}_{1}\right)$ and $\left(\mathbf{c}^{T}, \mathbf{A}_{2}, \mathbf{b}_{2}\right)$ given in our main result holds for the observable parts of the subsystems. For the unobservable part, $\mathbf{A}_{1} \mathbf{x}=\mathbf{A}_{2} \mathbf{x}$ for any $\mathbf{x} \in \operatorname{ker} \mathcal{O}_{n}^{1}$, as given in the Theorem 4.2 of [21].

Remark 3.4. Feedback well-posedness of BPAS is considered in [22]. The conditions given in [22] should reduce to the conditions given in our main result if the feedback terms are removed. However, since the setup in [22] is based on feedback terms (which involves controllability indices), such an equivalence is not readily obvious.

Remark 3.5. Theorem 3.1 of Thuan and Çamlıbel [23] provides sufficient conditions for a Filippov solution to be right unique and for each Filippov solution to be both forward and backward Carathéodory solution. (The definitions of Filippov solutions, backward Carathéodory solutions and Zeno Behavior are given in $[18,23]$.) For the case where both the pairs $\left(\mathbf{c}^{T}, \mathbf{A}_{1}\right)$ and $\left(\mathbf{c}^{T}, \mathbf{A}_{2}\right)$ are observable the Statement 5 of Theorem 3.1 in [23] can be written as follows

$\left[\mathcal{O}_{n+1}^{1}\right]=\mathbf{M}\left[\mathcal{O}_{n+1}^{2}\right], \quad\left[\begin{array}{c}f \\ \mathcal{O}_{n}^{1} \mathbf{b}_{1}\end{array}\right]=\mathbf{M}\left[\begin{array}{c}f \\ \mathcal{O}_{n}^{2} \mathbf{b}_{2}\end{array}\right]$,

where $\mathbf{M}$ is a lower left triangular matrix with positive diagonal entries, $\mathcal{O}_{k}^{i}$,s are as defined by Definition 2.1, and $f$ is a real scalar such that for $\mathbf{c}^{T} \mathbf{x}+f \geq 0$ the first mode is active and for $\mathbf{c}^{T} \mathbf{x}+f \leq 0$ the second mode is active. Similarly, Statement 6 of Theorem 3.1 in [23] can be written as follows.

$\left[\mathcal{O}_{n+1}^{1}\right] \mathbf{x}_{0}+\left[\begin{array}{c}f \\ \mathcal{O}_{n}^{1} \mathbf{b}_{1}\end{array}\right]=\mathbf{0} \Longrightarrow \mathbf{A}_{1} \mathbf{x}_{0}+\mathbf{b}_{1}=\mathbf{A}_{2} \mathbf{x}_{0}+\mathbf{b}_{2}$

Since $f=0$ in our setup, it can be easily shown that first equation in Statement 5 of Theorem 3.1 in [23] and positivity of the diagonal entries of $\mathbf{M}$ are equivalent to the first two items of our main result. 
Moreover, the second equation in Statement 5 of Theorem 3.1 in [23] is equivalent to Item 3 of our main result. Finally, Statement 6 of Theorem 3.1 in [23] is equivalent to Item 4 of our main result.

On the other hand, Implications C and D of Theorem 3.1 in [23] states that the conditions given above are sufficient conditions for every Filippov solution to be both forward and backward Carathéodory solution and every Filippov solution to be right unique, respectively. Consequently, in view of the results given in [23], our main result (Theorem 3.1) implies that Filippov solutions are right unique and they are both forward and backward Carathéodory solutions.

Remark 3.6. If $\Sigma_{0}$ is well-posed, then it is clear, by Example 3.2 and the proof of Theorem 3.1, that there exist affine spaces $\mathscr{H}_{k}, k=$ $1,2, \ldots, n-1$, each with dimension $n-k-1$, such that $\mathscr{H}_{n-1} \subset$ $\mathscr{H}_{n-2} \subset \cdots \subset \mathscr{H}_{1} \subset \mathcal{H}$ and

$$
\begin{aligned}
\mathscr{H}_{k}:= & \left\{\mathbf{x}_{0} \in \mathscr{H}_{k-1} \mid \mathbf{c}^{T} \mathbf{A}_{1}^{k-1}\left(\mathbf{A}_{1} \mathbf{x}_{0}+\mathbf{b}_{1}\right)\right. \\
= & \left.\mathbf{c}^{T} \mathbf{A}_{2}^{k-1}\left(\mathbf{A}_{2} \mathbf{x}_{0}+\mathbf{b}_{2}\right)=0\right\} .
\end{aligned}
$$

Furthermore, $\mathscr{H}_{k}$ divides $\mathscr{H}_{k-1}$ into two affine spaces $\mathscr{H}_{k}^{+}$and $\mathscr{H}_{k}^{-}$defined as follows.

$$
\begin{aligned}
\mathscr{H}_{k}^{+}:= & \left\{\mathbf{x}_{0} \in \mathscr{H}_{k-1} \mid \mathbf{c}^{T} \mathbf{A}_{1}^{k-1}\left(\mathbf{A}_{1} \mathbf{x}_{0}+\mathbf{b}_{1}\right)>0\right. \text { and } \\
& \left.\mathbf{c}^{T} \mathbf{A}_{2}^{k-1}\left(\mathbf{A}_{2} \mathbf{x}_{0}+\mathbf{b}_{2}\right)>0\right\}, \\
\mathscr{H}_{k}^{-}:= & \left\{\mathbf{x}_{0} \in \mathscr{H}_{k-1} \mid \mathbf{c}^{T} \mathbf{A}_{1}^{k-1}\left(\mathbf{A}_{1} \mathbf{x}_{0}+\mathbf{b}_{1}\right)<0\right. \text { and } \\
& \left.\mathbf{c}^{T} \mathbf{A}_{2}^{k-1}\left(\mathbf{A}_{2} \mathbf{x}_{0}+\mathbf{b}_{2}\right)<0\right\} .
\end{aligned}
$$

Note that if $\mathbf{x}_{0} \in \mathscr{H}_{1}^{+}\left(\mathscr{H}_{1}^{-}\right)$, then the solutions of $\Sigma_{0}$ can only arrive at $\mathbf{x}_{0}$ from $\mathscr{H}^{-}\left(\mathscr{H}^{+}\right)$and continue in $\mathscr{H}^{+}\left(\mathscr{H}^{-}\right)$. These solutions are both forward and backward Carathéodory solutions and $x_{0}$ is a switching point (any trajectory that arrives at $\mathbf{x}_{0}$ changes mode). However, if $\mathbf{x}_{0} \in \mathscr{H}_{2}^{+}\left(\mathscr{H}_{2}^{-}\right)$this is not necessarily true. In order to show the difference, suppose that $\mathbf{x}_{0} \in \mathscr{H}_{2}^{-}$and $\mathbf{x}(t)$ is a solution which starts from $\mathscr{H}^{+}$and continues in $\mathscr{H}^{+}$until $t=t_{1}$ and $\mathbf{x}\left(t_{1}\right)=\mathbf{x}_{0}$. Then, since the first derivative of $\mathbf{c}^{T} \mathbf{x}(t)$ is zero and the second derivative is negative at $t=t_{1}$, it follows that $\mathbf{c}^{T} \mathbf{x}(t)$ has a local maximum at $t=t_{1}$ and $\mathbf{c}^{T} \mathbf{x}(t)=0$. But, any small neighborhood of $\mathbf{c}^{T} \mathbf{x}\left(t_{1}\right)$ contains $\mathbf{c}^{T} \mathbf{x}(t)>0$ for $t<t_{1}$. This contradicts the fact that $\mathbf{c}^{T} \mathbf{x}\left(t_{1}\right)$ is a local maximum. Hence, the solutions of $\Sigma_{0}$ cannot arrive at $\mathbf{x}_{0} \in \mathscr{H}_{2}^{-}$from $\mathcal{H}^{+}$. Using similar reasoning with local minimums, it can be easily shown that the solutions of $\Sigma_{0}$ cannot arrive at $\mathbf{x}_{0} \in \mathcal{H}_{2}^{+}$from $\mathscr{H}^{-}$. On the other hand, there exist solutions $\mathbf{x}(t)$ of $\Sigma_{0}$ which starts from the second mode and continues in $\mathscr{H}^{-}$until $t=t_{1}$ and $\mathbf{x}\left(t_{1}\right)=\mathbf{x}_{0}$. Again $\mathbf{c}^{T} \mathbf{x}(t)$ has a local maximum at $t=t_{1}$. But, since the first derivative of $\mathbf{c}^{T} \mathbf{x}(t)$ is zero and the second derivative of $\mathbf{c}^{T} \mathbf{x}(t)$ is negative, $\mathbf{x}(t)$ will go back to $\mathscr{H}^{-}$and will not change mode.

We can summarize the observation given above as follows. If $\mathbf{x}_{0} \in \mathscr{H}_{2}^{-}\left(\mathscr{H}_{2}^{+}\right)$, then the solutions of $\Sigma_{0}$ can only arrive at $\mathbf{x}_{0}$ from $\mathscr{H}^{-}\left(\mathscr{H}^{+}\right)$and do not switch mode at $\mathbf{x}_{0}$. In each case, the solutions are both forward and backward Carathéodory solutions. Since forward (backward) Carathéodory solutions rule out left (right) Zeno behavior as noted in [18], it follows that well-posed BPAS considered in this work do not exhibit Zeno behavior.

It is clear from the development given above that the conditions of well-posedness require sign equalities for the derivatives of solutions for both modes on the hyperplane $\mathcal{H}$. On the other hand, the points on $\mathscr{H}$ where the derivatives of solutions for both modes are equal to the zero vector are equilibrium points of BPAS. At this stage, it is natural to look for the connections between the conditions of well-posedness and the equilibrium points of BPAS. Towards this end, suppose that $\mathbf{A}_{1}$ and $\mathbf{A}_{2}$ are nonsingular. Let
$\hat{\mathbf{b}}_{i}^{T}:=\left[\begin{array}{lllll}b_{i 2} & b_{i 3} & . . & \cdots & b_{i n}\end{array}\right]$ for $i=1,2$. Then, the last item of Theorem 3.1 can be written as follows.

$\operatorname{det}\left(\mathbf{A}_{11}\right)\left(b_{11}-\mathbf{y}_{1}^{T} \mathbf{A}_{11}^{-1} \hat{\mathbf{b}}_{1}\right)=b_{21}-\mathbf{y}_{2}^{T} \hat{\mathbf{b}}_{2}$,

where $\mathbf{y}_{2}$ is as defined in Eq. (5). As demonstrated in Example 3.2 this equality implies the existence of a point $\mathbf{x}_{0}^{*}:=\left[\hat{\mathbf{b}}_{i}^{T} 0\right]^{T}$ in $\mathscr{H} \cap \varsigma_{1} \cap \wp_{2}$. The existence of such a point(which may be the equilibrium point of BPAS) was pointed out in Remark 2.7. In what follows, we give the conditions under which $\mathbf{x}_{0}^{*}$ is the equilibrium point of BPAS (item 5 of Corollary 3.2) and also state the relations between the equilibrium points of the individual subsystems and equilibrium points of BPAS (items 1-4 of Corollary 3.2). Note that using the partitions of system matrices given in Eqs. (4) and (5) and the fact that $\mathbf{A}_{11}^{-1} \hat{\mathbf{b}}_{1}=\hat{\mathbf{b}}_{2}$ by item 3 of Theorem 3.1, it is easy to show that

$$
\begin{aligned}
-\mathbf{A}_{1}^{-1} \mathbf{b}_{1} & =\left[\begin{array}{c}
\frac{1}{\gamma} \mathbf{A}_{11}^{-1} \mathbf{z}_{1} b_{11}-\mathbf{A}_{11}^{-1} \hat{\mathbf{b}}_{1}-\frac{1}{\gamma} \mathbf{A}_{11}^{-1} \mathbf{z}_{1} \mathbf{y}_{1}^{T} \mathbf{A}_{11}^{-1} \hat{\mathbf{b}}_{1} \\
\frac{-1}{\gamma}\left(b_{11}-\mathbf{y}_{1}^{T} \mathbf{A}_{11}^{-1} \hat{\mathbf{b}}_{1}\right)
\end{array}\right] \\
= & {\left[\begin{array}{c}
\frac{1}{\gamma} \mathbf{A}_{11}^{-1} \mathbf{z}_{1}\left(b_{11}-\mathbf{y}_{1}^{T} \hat{\mathbf{b}}_{2}\right)-\hat{\mathbf{b}}_{2} \\
\frac{-1}{\gamma}\left(b_{11}-\mathbf{y}_{1}^{T} \hat{\mathbf{b}}_{2}\right)
\end{array}\right], }
\end{aligned}
$$

where $\gamma=a_{1 n}-\mathbf{y}_{1}^{T} \mathbf{A}_{11}^{-1} \mathbf{z}_{1} \in \mathbb{R}$. Similarly, we also have

$$
-\mathbf{A}_{2}^{-1} \mathbf{b}_{2}=\left[\begin{array}{c}
-\hat{\mathbf{b}}_{2} \\
\frac{-1}{k_{n}}\left(b_{21}-\mathbf{y}_{2}^{T} \hat{\mathbf{b}}_{2}\right)
\end{array}\right] .
$$

In view of the above development, we now state and prove the following result.

Corollary 3.2. Suppose that $\mathbf{A}_{1}$ and $\mathbf{A}_{2}$ are nonsingular for a wellposed BPAS as defined by Eqs. (2)-(5). Then, the following hold.

1. If $\gamma k_{n}>0$ and $\frac{-1}{\gamma}\left(b_{11}-\mathbf{y}_{1}^{T} \hat{\mathbf{b}}_{2}\right)>0$, then $\mathbf{x}_{0}=-\mathbf{A}_{1}^{-1} \mathbf{b}_{1}$ is the unique equilibrium point of $\Sigma_{0}$.

2. If $\gamma k_{n}>0$ and $\frac{-1}{\gamma}\left(b_{11}-\mathbf{y}_{1}^{T} \hat{\mathbf{b}}_{2}\right)<0$, then $\mathbf{x}_{0}=-\mathbf{A}_{2}^{-1} \mathbf{b}_{2}$ is the unique equilibrium point of $\Sigma_{0}$.

3. If $\gamma k_{n}<0$ and $\frac{-1}{\gamma}\left(b_{11}-\mathbf{y}_{1}^{T} \hat{\mathbf{b}}_{2}\right)>0$, then there are two equilibrium points $\left(-\mathbf{A}_{1}^{-1} \mathbf{b}_{1}\right.$ and $\left.-\mathbf{A}_{2}^{-1} \mathbf{b}_{2}\right)$ of $\Sigma_{0}$; one in $\mathcal{H}^{+}$and one in $\mathscr{H}^{-}$.

4. If $\gamma k_{n}<0$ and $\frac{-1}{\gamma}\left(b_{11}-\mathbf{y}_{1}^{T} \hat{\mathbf{b}}_{2}\right)<0$, then there are no equilibrium points of $\Sigma_{0}$.

5. If $b_{21}-\mathbf{y}_{2}^{T} \hat{\mathbf{b}}_{2}=b_{11}-\mathbf{y}_{1}^{T} \hat{\mathbf{b}}_{2}=0$, then $\left[\begin{array}{ll}-\hat{\mathbf{b}}_{2}^{T} & 0\end{array}\right]^{T}$ is the unique equilibrium point of $\Sigma_{0}$.

Proof. Recall that det $\left(\mathbf{A}_{11}\right)>0$. Since $\gamma k_{n}>0$, then $\gamma$ and $k_{n}$ have the same sign and are different from zero. If $\frac{-1}{\gamma}\left(b_{11}-\mathbf{y}_{1}^{T} \hat{\mathbf{b}}_{2}\right)>0$, then by Eqs. (6) and (8) it follows that $\frac{-1}{k_{n}}\left(b_{21}-\mathbf{y}_{2}^{T} \hat{\mathbf{b}}_{2}\right)>0$. This implies that at $\mathbf{x}_{0}=-\mathbf{A}_{1}^{-1} \mathbf{b}_{1}$, we get $\mathbf{A}_{1} \mathbf{x}_{0}+\mathbf{b}_{1}=\mathbf{0}$ and $\mathbf{c}^{T} \mathbf{x}_{0}>0$ (only the first mode is active). Thus, $-\mathbf{A}_{1}^{-1} \mathbf{b}_{1}$ is the unique equilibrium point of $\Sigma_{0}$.

Similarly, if $\frac{-1}{\gamma}\left(b_{11}-\mathbf{y}_{1}^{T} \hat{\mathbf{b}}_{2}\right)<0$, then $\frac{-1}{k_{n}}\left(b_{21}-\mathbf{y}_{2}^{T} \hat{\mathbf{b}}_{2}\right)<0$. Then at $\mathbf{x}_{0}=-\mathbf{A}_{2}^{-1} \mathbf{b}_{2}$, we get $\mathbf{A}_{2} \mathbf{x}_{0}+\mathbf{b}_{2}=\mathbf{0}$ and $\mathbf{c}^{T} \mathbf{x}_{0}<$ 0 (only the second mode is active). Thus, $-\mathbf{A}_{2}^{-1} \mathbf{b}_{2}$ is the unique equilibrium point of $\Sigma_{0}$. 
If $\gamma k_{n}<0$, then $\gamma$ and $k_{n}$ have the different signs and are different from zero. In this case, if $\frac{-1}{\gamma}\left(b_{11}-\mathbf{y}_{1}^{T} \hat{\mathbf{b}}_{2}\right)>0$, it follows that $\frac{-1}{k_{n}}\left(b_{21}-\mathbf{y}_{2}^{T} \hat{\mathbf{b}}_{2}\right)<0$. Then, at $\mathbf{x}_{0}=-\mathbf{A}_{1}^{-1} \mathbf{b}_{1}$, we get $\mathbf{A}_{1} \mathbf{x}_{0}+$ $\mathbf{b}_{1}=\mathbf{0}$ and $\mathbf{c}^{T} \mathbf{x}_{0}>0$. Similarly, at $\mathbf{x}_{0}=-\mathbf{A}_{2}^{-1} \mathbf{b}_{2}$, we get $\mathbf{A}_{2} \mathbf{x}_{0}+$ $\mathbf{b}_{2}=\mathbf{0}$ and $\mathbf{c}^{T} \mathbf{x}_{0}<0$. Consequently, there are two equilibrium points $\left(-\mathbf{A}_{1}^{-1} \mathbf{b}_{1}\right.$ and $\left.-\mathbf{A}_{2}^{-1} \mathbf{b}_{2}\right)$ of $\Sigma_{0}$; one in $\mathscr{H}^{+}$and one in $\mathscr{H}^{-}$.

On the other hand, if $\frac{-1}{\gamma}\left(b_{11}-\mathbf{y}_{1}^{T} \hat{\mathbf{b}}_{2}\right)<0$, then $\frac{-1}{k_{n}}\left(b_{21}-\right.$ $\left.\mathbf{y}_{2}^{T} \hat{\mathbf{b}}_{2}\right)>0$. Then, at $\mathbf{x}_{0}=-\mathbf{A}_{1}^{-1} \mathbf{b}_{1}$, we get $\mathbf{A}_{1} \mathbf{x}_{0}+\mathbf{b}_{1}=\mathbf{0}$ and $\mathbf{c}^{T} \mathbf{x}_{0}<0$ where the first mode is not allowed to be active. Similarly, at $\mathbf{x}_{0}=-\mathbf{A}_{2}^{-1} \mathbf{b}_{2}$, we get $\mathbf{A}_{2} \mathbf{x}_{0}+\mathbf{b}_{2}=\mathbf{0}$ and $\mathbf{c}^{T} \mathbf{x}_{0}>0$ where the second mode is not allowed to be active. Consequently, there are no equilibrium points of $\Sigma_{0}$.

Finally, if $\left(b_{21}-\mathbf{y}_{2}^{T} \hat{\mathbf{b}}_{2}\right)=\operatorname{det}\left(\mathbf{A}_{11}\right)\left(b_{11}-\mathbf{y}_{1}^{T} \mathbf{A}_{11}^{-1} \hat{\mathbf{b}}_{1}\right)=0$, then Eqs. (5) and (6) imply that $-\mathbf{A}_{2}^{-1} \mathbf{b}_{2}=-\mathbf{A}_{1}^{-1} \mathbf{b}_{1}=\left[\begin{array}{ll}-\hat{\mathbf{b}}_{2}^{T} & 0\end{array}\right]^{T}$. Let $\mathbf{x}_{0}=\left[\begin{array}{ll}-\hat{\mathbf{b}}_{2}^{T} & 0\end{array}\right]^{T}$. Then, it follows that $\mathbf{A}_{1} \mathbf{x}_{0}+\mathbf{b}_{1}=\mathbf{A}_{2} \mathbf{x}_{0}+\mathbf{b}_{2}=$ o. Consequently, $\left[\begin{array}{ll}-\hat{\mathbf{b}}_{2}^{T} & 0\end{array}\right]^{T}$ isthe unique equilibrium point of $\Sigma_{0}$.

\section{Conclusions and future work}

In this paper, well-posedness conditions (in the sense of forward Carathéodory solutions) of BPAS in $\mathbb{R}^{n}$, where the vector field is not necessarily continuous on the switching hyperplane $\mathcal{H}$, are investigated. It is shown that these conditions reduce to a set of algebraic equations and inequalities involving the subsystem matrices and affine terms. This yields a joint structure for wellposed BPAS triples $\left(\mathbf{c}^{T}, \mathbf{A}_{1}, \mathbf{b}_{1}\right)$ and $\left(\mathbf{c}^{T}, \mathbf{A}_{2}, \mathbf{b}_{2}\right)$ which can be checked by simple inspection. Furthermore, it is also demonstrated that well-posedness conditions for BPAS reduce to well-posedness conditions for BPLS, if $\mathbf{b}_{1}=\mathbf{b}_{2}=\mathbf{0}$. Thus, the results given in this work can be used for characterization of well-posed BPLS and BPAS with discontinuous vector fields. Finally, the conditions under which well-posed BPAS have one, or two or no equilibrium points are given.

The next step along this direction could be to investigate wellposedness conditions for multi-modal systems. Towards this end, conewise linear systems, which are investigated in [15] under the assumption that the vector field is continuous, can be considered. When the vector field is continuous, well-posedness is resolved. If we relax this assumption and allow the vector field to be discontinuous, the assessment of the conditions of well-posedness of conewise linear systems could be an interesting problem.

\section{Acknowledgments}

This work is supported in part by 2219 programme of The Scientific and Technological Research Council of Turkey, TUBITAK.

We are grateful to anonymous reviewers and Daniel Liberzon for their comments and suggestions which were most helpful in improving this work.

\section{Appendix}

Proof of Theorem 3.1. Since only one of the modes is active for the initial conditions in $\mathscr{H}^{-}\left(\mathscr{H}^{+}\right)$, there exist unique forward Carathéodory solutions which smoothly continue in $\mathscr{H}^{-}\left(\mathscr{H}^{+}\right)$. Therefore, in the proof, we only consider initial conditions in $\mathscr{H}$.
Let

$\mathbf{x}(0):=\mathbf{x}_{0}=\left[\begin{array}{lllllll}\gamma_{1} & \gamma_{2} & . . & . . & \gamma_{n-2} & \gamma_{n-1} & 0\end{array}\right]^{T} \in \operatorname{ker} \mathbf{c}^{T}$

where $\gamma_{1}, \gamma_{2}, \ldots, \gamma_{n-1}$ are arbitrary real numbers. Calculating the derivatives of $\mathbf{c}^{T} \mathbf{x}(t)$ at $t=0$ for both modes, we get

$$
\begin{aligned}
& \left.\frac{d}{\mathrm{~d} t}\left(\mathbf{c}^{T} \mathbf{x}\right)\right|_{t=0}=\left\{\begin{array}{l}
\mathbf{c}^{T}\left(\mathbf{A}_{1} \mathbf{x}_{0}+\mathbf{b}_{1}\right) \\
\mathbf{c}^{T}\left(\mathbf{A}_{2} \mathbf{x}_{0}+\mathbf{b}_{2}\right)
\end{array}\right\} \\
& =\left\{\begin{array}{c}
a_{n 1} \gamma_{1}+a_{n 2} \gamma_{2}+\cdots+a_{n, n-2} \gamma_{n-2}+a_{n, n-1} \gamma_{n-1}+b_{1 n} \\
\gamma_{n-1}+b_{2 n}
\end{array}\right\} .
\end{aligned}
$$

Let us fix $\gamma_{n-1}$ so that $\gamma_{n-1}+b_{2 n}=0$. Note that the sign of $a_{n 1} \gamma_{1}+$ $a_{n 2} \gamma_{2}+\cdots+a_{n, n-2} \gamma_{n-2}+a_{n, n-1} \gamma_{n-1}+b_{1 n}$ can be changed arbitrarily by appropriate choice of the real numbers $\gamma_{1}, \gamma_{2}, \ldots, \gamma_{n-2}$. This contradicts well-posedness. Thus, it is necessary that $a_{n 1}=$ $a_{n 2}=\cdots=a_{n, n-2}=0$ or equivalently $\operatorname{ker} \mathbf{c}^{T} \cap \operatorname{ker} \mathbf{c}^{T} \mathbf{A}_{1} \subset$ $\operatorname{ker} \mathbf{c}^{T} \cap \operatorname{ker} \mathbf{c}^{T} \mathbf{A}_{2}$. Since both modes are observable, it follows that $\operatorname{dim}\left(\operatorname{ker} \mathbf{c}^{T} \cap \operatorname{ker} \mathbf{c}^{T} \mathbf{A}_{1}\right)=\operatorname{dim}\left(\operatorname{ker} \mathbf{c}^{T} \cap \operatorname{ker} \mathbf{c}^{T} \mathbf{A}_{2}\right)$ which implies that $\operatorname{ker} \mathbf{c}^{T} \cap \operatorname{ker} \mathbf{c}^{T} \mathbf{A}_{1}=\operatorname{ker} \mathbf{c}^{T} \cap \operatorname{ker} \mathbf{c}^{T} \mathbf{A}_{2}$. Then, the derivatives of $\mathbf{c}^{T} \mathbf{x}(t)$ at $t=0$ for both modes are given as follows.

$\left.\frac{d}{\mathrm{~d} t}\left(\mathbf{c}^{T} \mathbf{x}\right)\right|_{t=0}=\left\{\begin{array}{c}\mathbf{c}^{T}\left(\mathbf{A}_{1} \mathbf{x}_{0}+\mathbf{b}_{1}\right) \\ \mathbf{c}^{T}\left(\mathbf{A}_{2} \mathbf{x}_{0}+\mathbf{b}_{2}\right)\end{array}\right\}=\left\{\begin{array}{c}a_{n, n-1} \gamma_{n-1}+b_{1 n} \\ \gamma_{n-1}+b_{2 n}\end{array}\right\}$.

Note that $a_{n, n-1} \neq 0$, because this contradicts observability. On the other hand, if $a_{n, n-1}<0$, then we can choose $\gamma_{n-1}>$ $\max \left\{\left|\frac{b_{1 n}}{a_{n, n-1}}\right|,\left|b_{2 n}\right|\right\}$ so that $a_{n, n-1} \gamma_{n-1}+b_{1 n}<0$ and $\gamma_{n-1}+$ $b_{2 n}>0$ which implies that there are no solutions in the sense of Carathéodory. Thus, it is necessary that $a_{n, n-1}>0$. In this case $a_{n, n-1} \gamma_{n-1}+b_{1 n}$ and $\gamma_{n-1}+b_{2 n}$ must have the same sign for all real numbers $\gamma_{n-1}$ in order to guarantee well-posedness. Consider the following sets in $\mathscr{H}$.

$$
\begin{aligned}
& \mathscr{H}_{11}:=\left\{\mathbf{x}_{0} \in \mathscr{H} \mid \mathbf{x}_{0}\right. \\
& \left.=\left[\begin{array}{lllllll}
\gamma_{1} & \gamma_{2} & . . & . & \gamma_{n-2} & \gamma_{n-1}-\frac{b_{1 n}}{a_{n, n-1}} & 0
\end{array}\right]^{T}\right\}, \\
& \mathscr{H}_{21}:=\left\{\mathbf{x}_{0} \in \mathscr{H} \mid \mathbf{x}_{0}\right. \\
& \left.=\left[\begin{array}{lllllll}
\gamma_{1} & \gamma_{2} & . . & . . & \gamma_{n-2} & \gamma_{n-1}-b_{2 n} & 0
\end{array}\right]^{T}\right\},
\end{aligned}
$$

where $\gamma_{1}, \gamma_{2}, \ldots, \gamma_{n-1}$ are arbitrary real numbers. If $\gamma_{n-1}=0$, then $\mathscr{H}_{11}$ and $\mathscr{H}_{21}$ are affine spaces (of dimension $n-2$ ) which describe the set of initial conditions where $\left.\frac{d}{d t}\left(\mathbf{c}^{T} \mathbf{x}\right)\right|_{t=0}=0$ for both modes. If these affine spaces are not equal, then we have three alternatives.

1. If $\gamma_{n-1}$ is chosen so that $\gamma_{n-1}>\max \left\{-b_{2 n},-\frac{b_{1 n}}{a_{n, n-1}}\right\}$, then $\left.\frac{d}{\mathrm{~d} t}\left(\mathbf{c}^{T} \mathbf{x}\right)\right|_{t=0}>0$ for both modes and there is a unique forward Carathéodory solution which smoothly continues in the first mode.

2. If $\gamma_{n-1}$ is chosen so that $\gamma_{n-1}<\min \left\{-b_{2 n},-\frac{b_{1 n}}{a_{n, n-1}}\right\}$, then $\left.\frac{d}{\mathrm{~d} t}\left(\mathbf{c}^{T} \mathbf{x}\right)\right|_{t=0}<0$ for both modes and there is a unique forward Carathéodory solution which smoothly continues in the second mode.

3. If $\gamma_{n-1}$ is chosen so that $\min \left\{-b_{2 n},-\frac{b_{1 n}}{a_{n, n-1}}\right\}<\gamma_{n-1}<$ $\max \left\{-b_{2 n},-\frac{b_{1 n}}{a_{n, n-1}}\right\}$, then the sign of $\left.\frac{d}{\mathrm{~d} t}\left(\mathbf{c}^{T} \mathbf{x}\right)\right|_{t=0}$ is different for each mode. If $a_{n, n-1} \gamma_{n-1}+b_{1 n}>0$ and $\gamma_{n-1}+b_{2 n}<0$, then there are two solutions which smoothly continue in separate modes. If $a_{n, n-1} \gamma_{n-1}+b_{1 n}<0$ and $\gamma_{n-1}+b_{2 n}>0$, then there are no solutions in the sense of Carathéodory. 
Consequently, it is necessary that $a_{n, n-1} b_{2 n}=b_{1 n}$ or equivalently $\mathscr{H}_{11}=\mathscr{H}_{21}$. Let $\mathscr{H}_{1}$ be an affine space (of dimension $n-2$ ) defined as $\mathscr{H}_{1}:=\mathscr{H}_{11}=\mathscr{H}_{21}$. Then, we have

$$
\begin{aligned}
& \mathscr{H}_{1}:=\left\{\mathbf{x}_{0} \in \mathscr{H} \mid \mathbf{x}_{0}=\left[\begin{array}{lllllll}
\gamma_{1} & \gamma_{2} & . . & . . & \gamma_{n-2} & -\frac{b_{1 n}}{a_{n, n-1}} & 0
\end{array}\right]^{T}\right\}, \\
& =\left\{\mathbf{x}_{0} \in \mathscr{H} \mid \mathbf{c}^{T}\left(\mathbf{A}_{1} \mathbf{x}_{0}+\mathbf{b}_{1}\right)=\mathbf{c}^{T}\left(\mathbf{A}_{2} \mathbf{x}_{0}+\mathbf{b}_{2}\right)=0\right\} .
\end{aligned}
$$

Consequently, the conditions

$a_{n 1}=a_{n 2}=\cdots=a_{n, n-2}=0$,

$a_{n, n-1} b_{2 n}=b_{1 n}$ and $a_{n, n-1}>0$

are necessary for the existence of a unique forward Carathéodory solution for each $\mathbf{x}_{0} \in \mathscr{H}$, except for $\mathbf{x}_{0} \in \mathscr{H}_{1}$.

Conversely, suppose that the conditions given in (9) hold. Then,

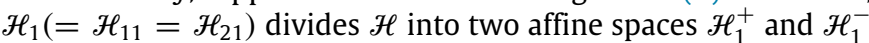
defined as follows.

$\mathscr{H}_{1}^{+}:=\left\{\mathbf{x}_{0} \in \mathscr{H} \mid \mathbf{c}^{T}\left(\mathbf{A}_{1} \mathbf{x}_{0}+\mathbf{b}_{1}\right)>0\right.$ and $\left.\mathbf{c}^{T}\left(\mathbf{A}_{2} \mathbf{x}_{0}+\mathbf{b}_{2}\right)>0\right\}$,

$\mathscr{H}_{1}^{-}:=\left\{\mathbf{x}_{0} \in \mathscr{H} \mid \mathbf{c}^{T}\left(\mathbf{A}_{1} \mathbf{x}_{0}+\mathbf{b}_{1}\right)<0\right.$ and $\left.\mathbf{c}^{T}\left(\mathbf{A}_{2} \mathbf{x}_{0}+\mathbf{b}_{2}\right)<0\right\}$.

Thus, for each initial condition $\mathbf{x}_{0} \in \mathscr{H}_{1}^{+}\left(\mathscr{H}_{1}^{-}\right)$there exists a unique forward Carathéodory solution which smoothly continues in the first (second) mode. Consequently, the conditions given in (9) are both necessary and sufficient conditions for the existence of a unique forward Carathéodory solution for each $\mathbf{x}_{0} \in \mathcal{H}$, except for $\mathbf{x}_{0} \in \mathscr{H}_{1}$. Note further that $\mathscr{H}_{1}^{+} \subset s_{1}$ and $\mathscr{H}_{1}^{-} \subset s_{2}$.

It is clear that for any initial condition in $\mathscr{H}_{1}$ we have to check the sign of the second derivative. Towards this end, let $\mathbf{x}_{0}=$ $\left[\begin{array}{lllllll}\gamma_{1} & \gamma_{2} & . . & . \cdot & \gamma_{n-2} & -b_{2 n} & 0\end{array}\right]^{T} \in \mathscr{H}_{1}$. In this case,we have

$$
\begin{aligned}
\left.\frac{d^{2}}{\mathrm{~d} t^{2}}\left(\mathbf{c}^{T} \mathbf{x}\right)\right|_{t=0} & =\left\{\begin{array}{c}
\mathbf{c}^{T} \mathbf{A}_{1}\left(\mathbf{A}_{1} \mathbf{x}_{0}+\mathbf{b}_{1}\right) \\
\mathbf{c}^{T} \mathbf{A}_{2}\left(\mathbf{A}_{2} \mathbf{x}_{0}+\mathbf{c}^{T} \mathbf{A}_{2} \mathbf{b}_{2}\right)
\end{array}\right\} \\
& =\left\{\begin{array}{c}
\mathbf{c}^{T} \mathbf{A}_{1}\left(\mathbf{A}_{1} \mathbf{x}_{0}+\mathbf{b}_{1}\right) \\
\gamma_{n-2}+b_{2, n-1}
\end{array}\right\} .
\end{aligned}
$$

Recall that $\mathbf{c}^{T} \mathbf{A}_{1}=\left[\begin{array}{llllll}0 & 0 & . \cdot & 0 & a_{n, n-1} & a_{n n}\end{array}\right]^{T}$. Let $\mathbf{e}_{i}$ be a vector with all entries equal to zero except for the ith entry which is equal to one. Then, $\mathbf{A}_{1} \mathbf{x}_{0}+\mathbf{b}_{1}$ can be written as follows.

$\mathbf{A}_{1} \mathbf{x}_{0}+\mathbf{b}_{1}$

$=\left[\begin{array}{llllll}\mathbf{e}_{1}^{T} \mathbf{A}_{1} \mathbf{x}_{0}+b_{11} & \mathbf{e}_{2}^{T} \mathbf{A}_{1} \mathbf{x}_{0}+b_{12} & \cdots & . \cdot & \mathbf{e}_{n-1}^{T} \mathbf{A}_{1} \mathbf{x}_{0}+b_{1, n-1} & -b_{1 n}+b_{1 n}\end{array}\right]^{T}$

$=\left[\begin{array}{llllll}\mathbf{e}_{1}^{T} \mathbf{A}_{1} \mathbf{x}_{0}+b_{11} & \mathbf{e}_{2}^{T} \mathbf{A}_{1} \mathbf{x}_{0}+b_{12} & \cdots & . \cdot & \mathbf{e}_{n-1}^{T} \mathbf{A}_{1} \mathbf{x}_{0}+b_{1, n-1} & 0\end{array}\right]^{T}$.

This implies that

$\mathbf{c}^{T} \mathbf{A}_{1}\left(\mathbf{A}_{1} \mathbf{x}_{0}+\mathbf{b}_{1}\right)$

$=a_{n, n-1}\left[a_{n-1,1} \gamma_{1}+\cdots+a_{n-1, n-2} \gamma_{n-2}-a_{n-1, n-1} b_{2, n}+b_{1, n-1}\right]$.

Let us fix $\gamma_{n-2}$ such that $\gamma_{n-2}+b_{2, n-1}=0$. Then, as in the previous case, the sign of $\mathbf{c}^{T} \mathbf{A}_{1}\left(\mathbf{A}_{1} \mathbf{x}_{0}+\mathbf{b}_{1}\right)$ can be changed arbitrarily by a suitable choice of $\gamma_{n-1,1}, \gamma_{n-1,2}, \ldots, \gamma_{n-1, n-3}$. Then, there will be cases where there are no solutions in the sense of Carathéodory. Hence, it is necessary that $a_{n-1,1}=a_{n-1,2}=\cdots=a_{n-1, n-3}=0$ or equivalently

$\operatorname{ker} \mathbf{c}^{T} \cap \operatorname{ker} \mathbf{c}^{T} \mathbf{A}_{2} \cap \operatorname{ker} \mathbf{c}^{T} \mathbf{A}_{2}^{2}=\operatorname{ker} \mathbf{c}^{T} \cap \operatorname{ker} \mathbf{c}^{T} \mathbf{A}_{1} \cap \operatorname{ker} \mathbf{c}^{T} \mathbf{A}_{1}^{2}$.

Then, the second derivative can be calculated as follows.

$$
\begin{aligned}
& \left.\frac{d^{2}}{\mathrm{~d} t^{2}}\left(\mathbf{c}^{T} \mathbf{x}\right)\right|_{t=0}=\left\{\begin{array}{l}
\mathbf{c}^{T} \mathbf{A}_{1}\left(\mathbf{A}_{1} \mathbf{x}_{0}+\mathbf{b}_{1}\right) \\
\mathbf{c}^{T} \mathbf{A}_{2}\left(\mathbf{A}_{2} \mathbf{x}_{0}+\mathbf{b}_{2}\right)
\end{array}\right\} \\
& =\left\{\begin{array}{c}
a_{n, n-1}\left(a_{n-1, n-2} \gamma_{n-2}+b_{1, n-1}-a_{n-1, n-1} b_{2 n}\right) \\
\gamma_{n-2}+b_{2, n-1}
\end{array}\right\} .
\end{aligned}
$$

Note that $a_{n-1, n-2} \neq 0$, because this contradicts observability. Also note that if $a_{n-1, n-2}<0$, then we can choose $\gamma_{n-2}>$ $\max \left\{\left|\frac{b_{1, n-1}-a_{n-1, n-1} b_{2 n}}{a_{n-1, n-2}}\right|,\left|b_{2 n}\right|\right\}$ so that $a_{n-1, n-2} \gamma_{n-2}+b_{1, n-1}-$ $a_{n-1, n-1} b_{2 n}<0$ and $\gamma_{n-1}+b_{2 n}>0$ which implies that there are no solutions in the sense of Carathéodory. Thus, it is also necessary that $a_{n-1, n-2}>0$. Let us now consider the following sets.

$\mathscr{H}_{12}:=\left\{\mathbf{x}_{0} \in \mathscr{H}_{1} \mid \mathbf{x}_{0}\right.$

$\left.=\left[\begin{array}{lllllll}\gamma_{1} & \gamma_{2} & . . & \cdots & \gamma_{n-3} & \gamma_{n-2}-\frac{b_{1, n-1}-a_{n-1, n-1} b_{2 n}}{a_{n-1, n-2}}-\frac{b_{1 n}}{a_{n, n-1}} & 0\end{array}\right]^{T}\right\}$

$\mathscr{H}_{22}:=\left\{\mathbf{x}_{0} \in \mathscr{H}_{1} \mid \mathbf{x}_{0}\right.$

$\left.=\left[\begin{array}{llllllll}\gamma_{1} & \gamma_{2} & . . & . . & \gamma_{n-3} & \gamma_{n-2}-b_{2, n-1} & -b_{2 n} & 0\end{array}\right]^{T}\right\}$,

where $\gamma_{1}, \gamma_{2}, \ldots, \gamma_{n-2}$ are arbitrary real numbers. Note that if $\gamma_{n-2}=0$, then $\mathscr{H}_{12}$ and $\mathscr{H}_{22}$ are affine spaces in $\mathscr{H}_{1}$ which describe the set of initial conditions where $\left.\frac{d}{\mathrm{~d} t}\left(\mathbf{c}^{T} \mathbf{x}\right)\right|_{t=0}=\left.\frac{d^{2}}{\mathrm{~d} t^{2}}\left(\mathbf{c}^{T} \mathbf{x}\right)\right|_{t=0}=$ 0 , for both modes. If these affine spaces are not equal, then since $a_{n, n-1}>0$, we have three alternatives.

1. If $\gamma_{n-2}$ is chosen so that

$\gamma_{n-2}>\max \left\{-b_{2, n-1},-\frac{b_{1, n-1}-a_{n-1, n-1} b_{2 n}}{a_{n-1, n-2}}\right\}$, then $\left.\frac{d^{2}}{\mathrm{~d} t^{2}}\left(\mathbf{c}^{T} \mathbf{x}\right)\right|_{t=0}$ $>0$ for both modes and there is a unique forward Carathéodory solution which smoothly continues in the first mode.

2. If $\gamma_{n-2}$ is chosen so that

$\gamma_{n-2}<\min \left\{-b_{2, n-1},-\frac{b_{1, n-1}-a_{n-1, n-1} b_{2 n}}{a_{n-1, n-2}}\right\}$, then $\left.\frac{d^{2}}{\mathrm{~d} t^{2}}\left(\mathbf{c}^{T} \mathbf{x}\right)\right|_{t=0}$ $<0$ for both modes and there is a unique forward Carathéodory solution which smoothly continues in the second mode.

3. If $\gamma_{n-2}$ is chosen so that $\min \left\{-b_{2, n-1},-\frac{b_{1, n-1}-a_{n-1, n-1} b_{2 n}}{a_{n-1, n-2}}\right\}<$ $\gamma_{n-2}<\max \left\{-b_{2, n-1},-\frac{b_{1, n-1}-a_{n-1, n-1} b_{2 n}}{a_{n-1, n-2}}\right\}$, then the sign of $\left.\frac{d^{2}}{d t^{2}}\left(\mathbf{c}^{T} \mathbf{x}\right)\right|_{t=0}$ is different for each mode. Then, either there are two solutions, (one for each mode) or there are no solutions in the sense of Carathéodory. Consequently, it is necessary that $b_{2, n-1}=\frac{b_{1, n-1}-a_{n-1, n-1} b_{2 n}}{a_{n-1, n-2}}$ or equivalently $\mathscr{H}_{12}=\mathscr{H}_{22}$. Let $\mathscr{H}_{2}$ be an affine space (of dimension $n-3$ ) defined as $\mathscr{H}_{2}:=\mathscr{H}_{12}=$ $\mathscr{H}_{22}$. Then, we get

$$
\begin{aligned}
\mathscr{H}_{2} & =\left\{\mathbf{x}_{0} \in \mathscr{H}_{1} \mid \mathbf{c}^{T} \mathbf{A}_{1}\left(\mathbf{A}_{1} \mathbf{x}_{0}+\mathbf{b}_{1}\right)=\mathbf{c}^{T} \mathbf{A}_{2}\left(\mathbf{A}_{2} \mathbf{x}_{0}+\mathbf{b}_{2}\right)=0\right\}, \\
& =\left\{\mathbf{x}_{0} \in \mathscr{H}_{1} \mid \mathbf{x}_{0}\right. \\
& \left.=\left[\begin{array}{llllllll}
\gamma_{1} & \gamma_{2} & . . & . & \gamma_{n-3} & -b_{2, n-1} & -b_{2 n} & 0
\end{array}\right]^{T}\right\},
\end{aligned}
$$

then the conditions

$$
\begin{aligned}
& a_{n-1,1}=a_{n-1,2}=\cdots=a_{n-1, n-3}=0, a_{n-1, n-2}>0, \\
& \text { and } b_{2, n-1}=\frac{b_{1, n-1}-a_{n-1, n-1} b_{2 n}}{a_{n-1, n-2}}
\end{aligned}
$$

are necessary for the existence of a unique forward Carathéodory solution for each $\mathbf{x}_{0} \in \mathscr{H}_{1}$, except for $\mathbf{x}_{0} \in \mathscr{H}_{2}$.

Conversely, if the conditions given in (10) hold, then $\mathscr{H}_{2}$ divides $\mathscr{H}_{1}$ into two affine spaces $\mathscr{H}_{2}^{+}$and $\mathscr{H}_{2}^{-}$defined as follows.

$$
\begin{aligned}
& \mathscr{H}_{2}^{+}:=\left\{\mathbf{x}_{0} \in \mathscr{H}_{1} \mid \mathbf{c}^{T} \mathbf{A}_{1}\left(\mathbf{A}_{1} \mathbf{x}_{0}+\mathbf{b}_{1}\right)>0\right. \\
& \text { and } \left.\mathbf{c}^{T} \mathbf{A}_{2}\left(\mathbf{A}_{2} \mathbf{x}_{0}+\mathbf{b}_{2}\right)>0\right\}, \\
& \mathcal{H}_{2}^{-}:=\left\{\mathbf{x}_{0} \in \mathscr{H}_{1} \mid \mathbf{c}^{T} \mathbf{A}_{1}\left(\mathbf{A}_{1} \mathbf{x}_{0}+\mathbf{b}_{1}\right)<0\right. \\
& \text { and } \left.\mathbf{c}^{T} \mathbf{A}_{2}\left(\mathbf{A}_{2} \mathbf{x}_{0}+\mathbf{b}_{2}\right)<0\right\} .
\end{aligned}
$$

Therefore, for each initial condition $\mathbf{x}_{0} \in \mathscr{H}_{2}^{+}\left(\mathscr{H}_{2}^{-}\right)$there exists unique forward Carathé odory solution which smoothly continues in the first (second) mode. Consequently, the conditions given 
above in expression (10) are both necessary and sufficient conditions for the existence of a unique forward Carathéodory solution for each $\mathbf{x}_{0} \in \mathscr{H}_{1}$, except for $\mathbf{x}_{0} \in \mathscr{H}_{2}$. Note further that $\mathscr{H}_{2}^{+} \subset s_{1}$ and $\mathscr{H}_{2}^{-} \subset \varsigma_{2}$.

Also note that the relation between $b_{2 n}, b_{2, n-1}$ and $b_{1 n}, b_{1, n-1}$ can be written compactly as follows.

$\left[\begin{array}{cc}a_{n-1, n-2} & a_{n-1, n-1} \\ 0 & a_{n, n-1}\end{array}\right]\left[\begin{array}{c}b_{2, n-1} \\ b_{2 n}\end{array}\right]=\left[\begin{array}{c}b_{1, n-1} \\ b_{1 n}\end{array}\right]$.

Furthermore, we have

$\mathbf{c}^{T} \mathbf{A}_{1}=\left[\begin{array}{lllll}0 & \cdots & 0 & a_{n, n-1} & a_{n n}\end{array}\right]$,

$\mathbf{c}^{T} \mathbf{A}_{1}^{2}=\left[\begin{array}{llllll}0 & \cdots & 0 & a_{n, n-1} a_{n-1, n-2} & \times & \times\end{array}\right]$

where $x$ denotes entries which are not calculated explicitly.

For the rest of the proof, we use mathematical induction, which essentially follows similar lines as above. Towards this end, suppose that $\bigcap_{r=0}^{s} \operatorname{ker} \mathbf{c}^{T} \mathbf{A}_{1}^{r}=\bigcap_{r=0}^{s} \operatorname{ker} \mathbf{c}^{T} \mathbf{A}_{2}^{r}$ for $s=1,2, \ldots, k<$ $n-2$, or equivalently $a_{n-i+1, j}=0$ for $i=1,2, \ldots, k$ and $j=1,2, \ldots, n-i-1$, hold. Also suppose that $a_{n-i+1, n-i}>0$ for $i=1,2, \ldots, k$ and

$$
\begin{aligned}
& {\left[\begin{array}{cccc}
a_{n-k+1, n-k} & a_{n-k+1, n-k+1} & \cdots & a_{n-k+1, n-1} \\
0 & a_{n-k+2, n-k+1} & \ddots & a_{n-k+2, n-1} \\
\vdots & \ddots & \ddots & \vdots \\
0 & \cdots & 0 & a_{n, n-1}
\end{array}\right]\left[\begin{array}{c}
b_{2, n-k+1} \\
b_{2, n-k+2} \\
\vdots \\
b_{2, n-1} \\
b_{2 n}
\end{array}\right]} \\
& =\left[\begin{array}{c}
b_{1, n-k+1} \\
b_{1, n-k+2} \\
\vdots \\
b_{1, n-1} \\
b_{1 n}
\end{array}\right] .
\end{aligned}
$$

Also let

$$
\mathbf{c}^{T} \mathbf{A}_{1}^{k}=\left[\begin{array}{lllllll}
0 & \cdots & 0 & a_{n, n-1} a_{n-1, n-2} \ldots a_{n-k+1, n-k} & \times & \cdots & \times
\end{array}\right] .
$$

Note that the preceding statements hold for $k=1,2$. Let $\mathbf{x}_{0} \in \mathscr{H}_{k}$ be an initial condition given as follows.

$\mathbf{x}_{0}=\left[\begin{array}{llllllll}\gamma_{1} & \gamma_{2} & \cdots & \gamma_{n-k-1} & -b_{2, n-k+1} & \cdots & -b_{2 n} & 0\end{array}\right]^{T}$,

where $\mathscr{H}_{k}$ is an affine space of dimension $n-k-1$. Then, for each $\mathbf{x}_{0} \in \mathscr{H}_{k}$, the derivatives of the trajectories are zero up to order $k$ for both modes and $\mathscr{H}_{k} \subset \mathscr{H}_{k-1} \subset \cdots \subset \mathscr{H}_{2} \subset \mathscr{H}_{1}$. Let us consider $(k+1)$ th derivative.

$$
\begin{aligned}
\left.\frac{d^{k+1}}{\mathrm{~d} t^{k+1}}\left(\mathbf{c}^{T} \mathbf{x}\right)\right|_{t=0} & =\left\{\begin{array}{c}
\mathbf{c}^{T} \mathbf{A}_{1}^{k}\left(\mathbf{A}_{1} \mathbf{x}_{0}+\mathbf{b}_{1}\right) \\
\mathbf{c}^{T} \mathbf{A}_{2}^{k}\left(\mathbf{A}_{2} \mathbf{x}_{0}+\mathbf{b}_{2}\right)
\end{array}\right\} \\
& =\left\{\begin{array}{c}
\mathbf{c}^{T} \mathbf{A}_{1}^{k}\left(\mathbf{A}_{1} \mathbf{x}_{0}+\mathbf{b}_{1}\right) \\
\gamma_{n-k-1}+b_{2, n-k}
\end{array}\right\} .
\end{aligned}
$$

Then, using Eqs. (11) and (12), $\mathbf{A}_{1} \mathbf{x}_{0}+\mathbf{b}_{1}$ can be written as in Box I. This implies that

$$
\begin{aligned}
\mathbf{c}^{T} \mathbf{A}_{1}^{k} & \left(\mathbf{A}_{1} \mathbf{x}_{0}+\mathbf{b}_{1}\right)=a_{n, n-1} a_{n-1, n-2} \ldots a_{n-k+1, n-k}\left[a_{n-k, 1} \gamma_{1}\right. \\
& +\cdots+a_{n-k, n-k-1} \gamma_{n-k-1}+b_{1, n-k}-a_{n-k, n-k} b_{2, n-k+1} \\
& \left.-\cdots-a_{n-k, n-1} b_{2 n}\right] .
\end{aligned}
$$

Let us fix $\gamma_{n-k-1}$ such that $\gamma_{n-k-1}+b_{2, n-k}=0$. Then, as in the previous cases, the sign of $b_{2, n-k}$ is fixed and the sign of $\mathbf{c}^{T} \mathbf{A}_{1}^{k}\left(\mathbf{A}_{1} \mathbf{x}_{0}+\mathbf{b}_{1}\right)$ can be changed arbitrarily by an appropriate choice of $\gamma_{1}, \gamma_{2}, \ldots, \gamma_{n-k-2}$. Hence, it is necessary that $a_{n-k, 1}=$ $a_{n-k, 2}=\cdots=a_{n-k, n-k-2}=0$ or equivalently $\bigcap_{r=0}^{k+1} \operatorname{ker} \mathbf{c}^{T} \mathbf{A}_{1}^{r}=$ $\bigcap_{r=0}^{k+1} \operatorname{ker} \mathbf{c}^{T} \mathbf{A}_{2}^{r}$. Then, $(k+1)$ th derivative can be calculated as in Box II.
Note that $a_{n-k, n-k-1} \neq 0$, because this contradicts observability. Furthermore, if $a_{n-k, n-k-1}<0$, then we can choose $\gamma_{n-k-1}>$ $\max \left\{\left|\frac{b_{1, n-k}-a_{n-k, n-k} b_{2, n-k+1}-\cdots-a_{n-k, n-1} b_{2 n}}{a_{n-k, n-k-1}}\right|,\left|b_{2, n-k}\right|\right\}$ so that

$$
\left[a_{n-k, n-k-1} \gamma_{n-k-1}+b_{1, n-k}-a_{n-k, n-k} b_{2, n-k+1}\right.
$$$$
\left.-\cdots-a_{n-k, n-1} b_{2 n}\right]<0
$$

and $\gamma_{n-k-1}+b_{2, n-k}>0$, which implies that there are no solutions in the sense of Carathéodory. Thus, it is necessary that $a_{n-k, n-k-1}>$ 0 , which implies that $a_{n, n-1} a_{n-1, n-2} \ldots a_{n-k, n-k-1}>0$. Note that

$$
\begin{aligned}
& {\left[a_{n-k, n-k-1} \gamma_{n-k-1}+b_{1, n-k}-a_{n-k, n-k} b_{1, n-k+1}\right.} \\
& \left.\quad-\cdots-a_{n-k, n-1} b_{1 n}\right]
\end{aligned}
$$

and $\gamma_{n-k-1}+b_{2, n-1}$ must have same sign for the existence of a unique forward Carathéodory solution starting from $\mathbf{x}_{0}$. Let us now consider the following sets of equations in Box III, where $\gamma_{1}, \gamma_{2}, \ldots, \gamma_{n-k-1}$ are arbitrary real numbers. Note that if $\gamma_{n-k-1}=0$, then $\mathscr{H}_{1, k+1}$ and $\mathscr{H}_{2, k+1}$ are affine spaces in $\mathscr{H}_{k}$ which describe the set of initial conditions where $\left.\frac{d}{\mathrm{~d} t}\left(\mathbf{c}^{T} \mathbf{x}\right)\right|_{t=0}=$ $\left.\frac{d^{2}}{\mathrm{~d} t^{2}}\left(\mathbf{c}^{T} \mathbf{x}\right)\right|_{t=0}=\cdots=\left.\frac{d^{k+1}}{\mathrm{~d} t^{k+1}}\left(\mathbf{c}^{T} \mathbf{x}\right)\right|_{t=0}=0$, for first and second modes, respectively. If these affine spaces are not equal, then we have three alternatives.

1. If $\gamma_{n-k-1}$ is chosen so that

$\gamma_{n-k-1}>\max \left\{-b_{2, n-k},-\frac{b_{1, n-k}-a_{n-k, n-k} b_{2, n-k+1}-\cdots-a_{n-k, n-1} b_{2 n}}{a_{n-k, n-k-1}}\right\}$, then $\left.\frac{d^{k+1}}{d t^{k+1}}\left(\mathbf{c}^{T} \mathbf{x}\right)\right|_{t=0}>0$ for both modes and there is a unique forward Carathéodory solution which smoothly continues in the first mode.

2. If $\gamma_{n-k-1}$ is chosen so that

$\gamma_{n-k-1}<\min \left\{-b_{2, n-k},-\frac{b_{1, n-k}-a_{n-k, n-k} b_{2, n-k+1}-\cdots-a_{n-k, n-1} b_{2 n}}{a_{n-k, n-k-1}}\right\}$, then $\left.\frac{d^{k+1}}{\mathrm{~d} t^{k+1}}\left(\mathbf{c}^{T} \mathbf{x}\right)\right|_{t=0}<0$ for both modes and there is a unique forward Caratheodory solution which smoothly continues in the second mode.

3. If $\gamma_{n-k-1}$ is chosen so that

$$
\begin{aligned}
& \min \left\{-b_{2, n-k},-\frac{b_{1, n-k}-a_{n-k, n-k} b_{2, n-k+1}-\cdots-a_{n-k, n-1} b_{2 n}}{a_{n-k, n-k-1}}\right\} \\
& <\gamma_{n-k-1} \\
& <\max \left\{-b_{2, n-k},-\frac{b_{1, n-k}-a_{n-k, n-k} b_{2, n-k+1}-\cdots-a_{n-k, n-1} b_{2 n}}{a_{n-k, n-k-1}}\right\},
\end{aligned}
$$

then the sign of $\left.\frac{d^{k+1}}{d t^{k+1}}\left(\mathbf{c}^{T} \mathbf{x}\right)\right|_{t=0}$ is different for each mode. Then, either there are two solutions, (one in each mode) or there are no solutions in the sense of Carathéodory. Consequently, it is necessary that $b_{2, n-k}=\frac{b_{1, n-k}-a_{n-k, n-k} b_{2, n-k+1}-\cdots-a_{n-k, n-1} b_{2 n}}{a_{n-k, n-k-1}}$ or equivalently $\mathscr{H}_{1, k+1}=\mathscr{H}_{2, k+1}$. Let $\mathscr{H}_{k+1}$ be an affine space (of dimension $n-k-2$ ) defined as $\mathscr{H}_{k+1}:=\mathscr{H}_{1, k+1}=\mathscr{H}_{2, k+1}$. Then, we have given equations in Box IV.

Furthermore, the equation above also implies that $b_{1, n-k}=$ $a_{n-k, n-k-1} b_{2, n-k}+a_{n-k, n-k} b_{2, n-k+1}+\cdots+a_{n-k, n-1} b_{2 n}$. Therefore, the conditions

$$
\begin{gathered}
\bigcap_{r=0}^{k+1} \operatorname{ker} \mathbf{c}^{T} \mathbf{A}_{1}^{r}=\bigcap_{r=0}^{k+1} \operatorname{ker} \mathbf{c}^{T} \mathbf{A}_{2}^{r}, \quad a_{n-k, n-k-1}>0 \text { and } \\
b_{1, n-k}=a_{n-k, n-k-1} b_{2, n-k}+a_{n-k, n-k} b_{2, n-k+1} \\
\quad+\cdots+a_{n-k, n-1} b_{2 n} .
\end{gathered}
$$

are necessary for the existence of a unique forward Carathéodory solution for each $\mathbf{x}_{0} \in \mathscr{H}_{k}$, except $\mathbf{x}_{0} \in \mathscr{H}_{k+1}$.

Conversely, if the conditions given in expression (13) hold, then $\mathscr{H}_{k+1}$ divides $\mathscr{H}_{k}$ into two affine spaces $\mathscr{H}_{k+1}^{+}$and $\mathscr{H}_{k+1}^{-}$given as fol- 


$$
\begin{aligned}
\mathbf{A}_{1} \mathbf{x}_{0}+\mathbf{b}_{1} & =\left[\begin{array}{llllll}
\mathbf{e}_{1}^{T} \mathbf{A}_{1} \mathbf{x}_{0}+b_{11} & . . & \mathbf{e}_{n-k-1}^{T} \mathbf{A}_{1} \mathbf{x}_{0}+b_{1, n-k} & -b_{1, n-k+1}+b_{1, n-k+1} & . . & -b_{1 n}+b_{1 n}
\end{array}\right]^{T} \\
& =\left[\begin{array}{llllll}
\mathbf{e}_{1}^{T} \mathbf{A}_{1} \mathbf{x}_{0}+b_{11} & . \cdot & \mathbf{e}_{n-k-1}^{T} \mathbf{A}_{1} \mathbf{x}_{0}+b_{1, n-k} & 0 & . \cdot & 0
\end{array}\right]^{T} .
\end{aligned}
$$

Box I.

$$
\begin{aligned}
\left.\frac{d^{k+1}}{\mathrm{~d} t^{k+1}}\left(\mathbf{c}^{T} \mathbf{x}\right)\right|_{t=0} & =\left\{\begin{array}{l}
\mathbf{c}^{T} \mathbf{A}_{1}^{k}\left(\mathbf{A}_{1} \mathbf{x}_{0}+\mathbf{b}_{1}\right) \\
\mathbf{c}^{T} \mathbf{A}_{2}^{k}\left(\mathbf{A}_{2} \mathbf{x}_{0}+\mathbf{b}_{2}\right)
\end{array}\right\} \\
& =\left\{\begin{array}{c}
a_{n, n-1} a_{n-1, n-2} \cdots a_{n-k+1, n-k}\left[\begin{array}{c}
\left.a_{n-k, n-k-1} \gamma_{n-k-1}+b_{1, n-k}-a_{n-k, n-k} b_{2, n-k+1}-\cdots-a_{n-k, n-1} b_{2 n}\right] \\
\gamma_{n-k-1}+b_{2, n-k}
\end{array}\right\} .
\end{array}\right.
\end{aligned}
$$

Box II.

$$
\begin{aligned}
& \mathscr{H}_{1, k+1}:=\left\{\mathbf{x}_{0} \in \mathscr{H}_{k} \mid \mathbf{x}_{0}=\left[\begin{array}{lllllll}
\gamma_{1} & \gamma_{2} & . . & \gamma_{n-k-2} & \gamma_{n-k-1}-\frac{b_{1, n-k}-a_{n-k, n-k} b_{2, n-k+1}-\cdots-a_{n-k, n-1} b_{2 n}}{a_{n-k, n-k-1}} & \frac{-b_{1 n}}{a_{n, n-1}} & 0
\end{array}\right]^{T}\right\},
\end{aligned}
$$

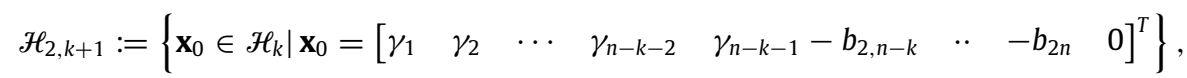

Box III.

$$
\begin{aligned}
& \mathscr{H}_{k+1}=\left\{\mathbf{x}_{0} \in \mathscr{H}_{k} \mid \mathbf{c}^{T} \mathbf{A}_{1}^{k}\left(\mathbf{A}_{1} \mathbf{x}_{0}+\mathbf{b}_{1}\right)=\mathbf{c}^{T} \mathbf{A}_{2}^{k}\left(\mathbf{A}_{2} \mathbf{x}_{0}+\mathbf{b}_{2}\right)=0\right\} \\
& \quad=\left\{\mathbf{x}_{0} \in \mathscr{H}_{k} \mid \mathbf{x}_{0}=\left[\begin{array}{llllllll}
\gamma_{1} & \gamma_{2} & \cdots & \gamma_{n-k-2} & -\frac{b_{1, n-k}-a_{n-k, n-k} b_{2, n-k+1}-\cdots-a_{n-k, n-1} b_{2 n}}{a_{n-k, n-k-1}} & . \cdot & -\frac{b_{1 n}}{a_{n, n-1}} & 0
\end{array}\right]^{T}\right\} .
\end{aligned}
$$

Box IV.

lows.

$$
\begin{aligned}
\mathscr{H}_{k+1}^{+}:=\{ & \mathbf{x}_{0} \in \mathcal{H}_{k} \mid \mathbf{c}^{T} \mathbf{A}_{1}^{k}\left(\mathbf{A}_{1} \mathbf{x}_{0}+\mathbf{b}_{1}\right)>0 \text { and } \\
& \left.\mathbf{c}^{T} \mathbf{A}_{2}^{k}\left(\mathbf{A}_{2} \mathbf{x}_{0}+\mathbf{b}_{2}\right)>0\right\}, \\
\mathscr{H}_{k+1}^{-}= & \left\{\mathbf{x}_{0} \in \mathscr{H}_{k} \mid \mathbf{c}^{T} \mathbf{A}_{1}^{k}\left(\mathbf{A}_{1} \mathbf{x}_{0}+\mathbf{b}_{1}\right)<0\right. \text { and } \\
& \left.\mathbf{c}^{T} \mathbf{A}_{2}^{k}\left(\mathbf{A}_{2} \mathbf{x}_{0}+\mathbf{b}_{2}\right)<0\right\} .
\end{aligned}
$$

This implies that there exists a unique forward Carathéodory solution for each $\mathbf{x}_{0} \in \mathscr{H}_{k}$, except $\mathbf{x}_{0} \in \mathscr{H}_{k+1}$. Thus, the conditions given in expression (13) are both necessary and sufficient for the existence of a unique forward Carathéodory solution for each $\mathbf{x}_{0} \in \mathscr{H}_{k}$, except $\mathbf{x}_{0} \in \mathcal{H}_{k+1}$. Then, by induction it follows that items 1,2 and 3 of Theorem 3.1 are necessary and sufficient for the existence of a unique forward Carathéodory solution for each $\mathbf{x}_{0} \in \mathbb{R}^{n}$, except for $\mathscr{H}_{n-1}$. Note further that $\mathscr{H}_{i}^{+} \subset s_{1}$ and $\mathscr{H}_{i}^{-} \subset s_{2}$ for $i=1,2, \ldots, n-1$. Moreover, $\mathscr{H}_{n-1}$ has zero dimension or consists of a single point $\mathbf{x}_{0}^{*}=\left[\begin{array}{llllll}-b_{22} & -b_{23} & \cdots & -b_{2, n-1} & -b_{2 n} & 0\end{array}\right]^{T}$. Recall that for this initial condition, all the derivatives up to order $n-1$ are zero for both modes. Calculating the $n$th derivative of $\left(\mathbf{c}^{T} \mathbf{x}\right)$ for both modes at $t=0$, we get

$$
\begin{aligned}
& \left.\frac{d^{n}}{\mathrm{~d} t^{n}}\left(\mathbf{c}^{T} \mathbf{x}\right)\right|_{t=0}=\left\{\begin{array}{l}
\mathbf{c}^{T} \mathbf{A}_{1}^{n-1}\left(\mathbf{A}_{1} \mathbf{x}_{0}^{*}+\mathbf{b}_{1}\right) \\
\mathbf{c}^{T} \mathbf{A}_{2}^{n-1}\left(\mathbf{A}_{2} \mathbf{x}_{0}^{*}+\mathbf{b}_{2}\right)
\end{array}\right\} \\
& =\left\{\begin{array}{c}
a_{n, n-1} a_{n-1, n-2} \ldots a_{21}\left[b_{11}-a_{11} b_{22}-\cdots-a_{1, n-1} b_{2 n}\right] \\
b_{21}-\sum_{j=1}^{n-1} k_{j} b_{2, j+1}
\end{array}\right\} .
\end{aligned}
$$

Note that $\mathbf{c}^{T} \mathbf{A}_{i}^{k-1}\left(\mathbf{A}_{i} \mathbf{x}_{0}^{*}+\mathbf{b}_{i}\right)=0$ for $i=1,2$ and $k=1,2, \ldots, n-$ 1. Therefore, if

$$
\begin{aligned}
& a_{n, n-1} a_{n-1, n-2} \ldots a_{21}\left[b_{11}-a_{11} b_{22}-\cdots-a_{1, n-1} b_{2 n}\right] \\
& \quad=b_{21}-\sum_{j=1}^{n-1} k_{j} b_{2, j+1}
\end{aligned}
$$

then, since both modes are observable, it follows that $\left(\mathbf{A}_{1} \mathbf{x}_{0}^{*}+\mathbf{b}_{1}\right)$ $=\left(\mathbf{A}_{2} \mathbf{x}_{0}^{*}+\mathbf{b}_{2}\right)$. This means that the solutions for both modes are the same. Conversely, if the solutions for both modes are the same, then $\left(\mathbf{A}_{1} \mathbf{x}_{0}^{*}+\mathbf{b}_{1}\right)=\left(\mathbf{A}_{2} \mathbf{x}_{0}^{*}+\mathbf{b}_{2}\right)$ which implies that Eq. (14) holds. Consequently, for any $\mathbf{x}_{0}^{*} \in \varsigma_{1} \cap \S_{2}$ solutions in both modes are the same and this concludes the proof.

\section{References}

[1] A.F. Filippov, Differential Equations With Discontinuous Right Hand Sides, in: Mathematics and Its Applications, Prentice-Hall, Dordrecht, The Netherlands, 1988.

[2] M. di Bernardo, C.J. Budd, A.R. Champneys, Kowalcyzk, Piecewise-smooth Dynamical Systems, Springer-Verlag, 2008.

[3] J. Cortes, Discontinuous dynamic systems, IEEE Control Syst. Mag. 28 (3) (2008)

[4] R. Goebel, R.G. Sanfelice, A.R. Teel, Hybrid dynamic systems, IEEE Control Syst. Mag. 29 (2) (2009) 699-724.

[5] D. Liberzon, Switching in Systems and Control, Birkhauser, Boston, 2003.

[6] M.K. Camlıbel, W.P.M.H. Heemels, J.M. Schumacher, A full characterization of stabilizability of bimodal piecewise linear systems with scalar inputs, Automatica 44 (2008) 1261-1267.

[7] M.K. Çamlıbel, W.P.M.H. Heemels, J.M. Schumacher, Algebraic necessary and sufficient conditions for the controllability of conewise linear systems, IEEE Trans. Automat. Control 53 (3) (2008) 762-774.

[8] V. Eldem, G. Şahan, Structure and stability of bimodal systems in R3: Part I, Appl. Comput. Math. 13 (2) (2014) 206-229.

[9] X. Liu, H. Lin, M. Chen Ben, Null controllability of planar bimodal piecewise linear systems, Internat. J. Control 84 (4) (2011) 766-782.

[10] Sun Zhendong, Stability of piecewise linear systems revisited, Ann. Rev. Control 34 (2) (2010) 221-223.

[11] V. Carmona, F. Fernández-Sánchez, A.E. Teruel, Existence of a reversible T-point heteroclinic cycle in a piecewise linear version of the Michelson system, SIAM J. Appl. Dyn. Syst. 7 (2008) 1032-1048.

[12] S. Huan, L. Qingdu, X.S. Yang, Chaos in three-dimensional hybrid systems and design of chaos generators, Nonlinear Dyn. 69 (2012) 1915-1927.

[13] Jun-ichi Imura, Kenji Kashima, Masami Kusano, Piecewise affine systems approach to control of biological networks, Philos. Trans. R. Soc. Lond. Ser. A Math. Phys. Eng. Sci. 368 (1930) (2010) 4977-4993.

[14] V. Carmona, Emilio Freire, Enrique Ponce, Francisco Torres, Bifurcation of invariant cones in piecewise linear homogeneous systems, Int. J. Bifur. Chaos 15 (8) (2005) 2469-2484.

[15] M.K. Çamlıbel, J.S. Pang, J. Shen, Conewise linear systems: Non-zenoness and observability, SIAM J. Control Optim. 45 (5) (2006) 1769-1800.

[16] C. Georgescu, B. Brogliato, V. Acary, Switching, relay and complementarity systems: A tutorial on their well-posedness and relationships, Physica D 241 (2012) 1985-2002. 
[17] V. Utkin, Sliding Mode Control: Mathematical Tools, Design and Applications, in: Nonlinear and Optimal Control Theory Lecture Notes in Mathematics, vol. 1932, Springer, 2008, pp. 289-347.

[18] M.K. Çamlıbel, Well-posed bimodal piecewise linear systems do not exhibit zeno behavior, in: Proc. of the 17th IFAC Congress Seoul Korea, 2008, pp. 7973-7978.

[19] L.Q. Thuan, M.K. Çamlıbel, Continuous piecewise affine dynamic systems do not exhibit zeno behavior, IEEE Trans. Automat. Control 56 (8) (2011) 1932-1936.

[20] Y. Iwatani, S. Hara, Stability tests and stabilization for piecewise linear systems based on poles and zeros of subsystems, Automatica 42 (2006) 1685-1695.
[21] Jun-ichi Imura, A. van der Schaft, Characterization of well-posedness of piecewise-linear systems, IEEE Trans. Automat. Control 45 (2000) 1600-1619.

[22] Jun-ichi Imura, Classification and stabilizability analysis of bimodal piecewise affine systems, Internat. J. Robust Nonlinear Control 12 (2002) 897-926.

[23] L.O. Thuan, M.K. Camlibel, On the existence, uniqueness and the nature of Carathéodory and Filippov solutions for bimodal piecewise affine systems, Syst. Control Lett. 68 (2014) 76-85.

[24] A.J. Van der Schaft, J.M. Schumacher, An Introduction to Hybrid Dynamical Systems, in: Lecture Notes in Control and Information Sciences, vol. 251, Springer, Berlin, 2000.

[25] C.Z. Wu, K.L. Teo, V. Rehbock, Well-posednes of bimodal state-based switched systems, Appl. Math. Lett. 21 (2008) 835-839. 